\title{
POLYANALYTIC FUNCTIONS WITH EXCEPTIONAL VALUES
}

\section{P. KRAJKIEWICZ}

ABSTRACT. Let $f(z)=\sum_{k=0}^{n} \bar{z}^{k} f_{k}(z)$ where the functions $f_{0}, f_{1}, \cdots, f_{n}$ are analytic on some annular neighborhood $A$ of the point $\infty$ and $f_{n} \equiv 1$ on $A$ and $\bar{z}$ denotes the complex conjugate of $z$. If $f$ does not vanish on $A$ it is shown that the functions $f_{0}, f_{1}, \cdots, f_{n-1}$ have a nonessential isolated singularity at the point infinity.

1. Introduction. If $\Gamma$ denotes the finite complex plane, a function $f: \Gamma \rightarrow \Gamma$ is said to be polyentire or $(n+1)$ entire if and only if there exist $(n+1) \geq 1$ entire functions $f_{0}, f_{1}, \cdots, f_{n}$ such that $f(z)=f_{0}(z)+\bar{z} f_{1}(z)+\bar{z}^{2} f_{2}(z)+\cdots+$ $\bar{z}^{n} f_{n}(z)$ for all $z \in \Gamma$, where $\bar{z}$ denotes the complex conjugate of $z$. A function $f$ is said to be bientire if and only if $f$ is $(n+1)$ entire with $n=1$. More generally if $A$ is any nonempty subset of $\Gamma$, a function $f: A \rightarrow \Gamma$ is said to be polyanalytic on $A$ or $(n+1)$ analytic on $A$ if and only if there exist $(n+1) \geq 1$ functions $f_{0}, f_{1}, \cdots, f_{n}$ analytic on $A$ such that

$$
f(z)=\sum_{k=0}^{n} \bar{z}^{k} f_{k}(z),
$$

for all $z \in A$, where $\bar{z}$ denotes the complex conjugate of $z$. A function $f$ is said to be bianalytic on $A$ if and only if $f$ is $(n+1)$ analytic on $A$ with $n=1$.

Now let $f$ be polyanalytic on some nonempty subset $A$ of $\Gamma$ and let $z_{0}$ be an arbitrary complex number, finite or infinite. Then the point $z_{0}$ is said to be an isolated singularity of $f$ if and only if there is some neighborhood $N$ of $z_{0}$ so that $N-\left\{z_{0}\right\} \subseteq A$.

We now need the following uniqueness result.

Lemma (1.1). If $f$ is polyanalytic on a nonempty open subset $G$ of $\Gamma$ and if $f$ is represented on $G$ by equation (1.1), then the functions $f_{0}, f_{1}, \cdots, f_{n}$ in equation (1.1) are uniquely determined on $G$ by $\%$

Proof. It suffices to show that if $f \equiv 0$ on $G$, then $f_{k} \equiv 0$ on $G$ for $k=$ $0,1, \cdots, n$. If we introduce the operator $\partial / \partial \bar{z}=1 / 2(\partial / \partial x+i \partial / \partial y)$, it is easy to see that

Received by the editors March 26, 1973 and, in revised form, October 31, 1973. AMS (MOS) subject classifications (1970). Primary 30A94; Secondary 30A70.

Key words and phrases. Polyanalytic functions, polyentire functions, essential isolated singularity, exceptional values, Picard's big theorem. 


$$
\frac{\partial f}{\partial \bar{z}}(z)=\sum_{k=0}^{n} k \bar{z}^{k-1} f_{k}(z),
$$

for $z \in G$. If $f \equiv 0$ on $G$, it then follows that $\partial^{k} f / \partial \bar{z}^{k} \equiv 0$ on $G$ for $k=0,1, \cdots, n$. From these $(n+1) \geq 1$ equations we deduce that $f_{k} \equiv 0$ on $G$ for $k=0,1, \cdots, n$. This proves the result.

In view of the above uniqueness result, the following definitions are not ambiguous. Let $f$ be a polyanalytic function with an isolated singularity at point $z_{0}$. Then there is some neighborhood $N$ of $z_{0}$ so that $f$ is represented on $N-$ $\left\{z_{0}\right\}$ by (1.1). This representation is unique and the functions $f_{0}, f_{1}, \cdots, f_{n}$ in (1.1) all have an isolated singularity at the point $z_{0}$. The point $z_{0}$ is said to be an essential isolated singularity of $f$ if and only if the point $z_{0}$ is an essential isolated singularity of at least one of the functions $f_{0}, f_{1}, \cdots, f_{n}$. The point $z_{0}$ is said to be a nonessential isolated singularity of $f$ if and only if the point $z_{0}$ is a nonessential isolated singularity of every one of the functions $f_{0}, f_{1}, \cdots, f_{n}$.

Next let $f$ be a polyanalytic function with an isolated singularity at a point $z_{0}$ and let $\alpha$ be a finite complex number. Then $\alpha$ is said to be an exceptional value for $f$ and $z_{0}$ if and only if there is some neighborhood $N$ of $z_{0}$ so that $f-\alpha$ never vanishes on $N-\left\{z_{0}\right\}_{0}$

In [1], M. B. Balk undertook the study of polyentire functions which admit an exceptional value at the point infinity. The author, by utilizing H. Cartan's theory of meromorphic curves [3], succeeded, in a long and complicated argument, in establishing the following elegant result.

If $f$ is a polyentire function which admits the exceptional value zeto at the point infinity, then there exists an entire function $h$ and a polynomial $P(z, \bar{z})$ in $z$ and $\bar{z}$ such that

$$
f(z)=e^{b(z)} P(z, \bar{z}), \text { for all } z \in \Gamma .
$$

Subsequently in [6], the author, by a rather elementary argument utilizing the theory of quasinormal families of analytic functions, obtained a similar characterization of bianalytic functions which admit the exceptional value zero at an isolated singularity, finite or infinite.

The results cited above in [1] and [6] suggest a study of polyanalytic functions which admit an exceptional value at an isolated singularity.

Before stating our main result, it will be convenient to introduce the following notation. Let $0<R \leq+\infty$ and let $z_{0}$ be an arbitrary complex number, finite or infinite. We now define $A\left(z_{0}, R\right)$ as follows. If $z_{0}$ is finite, we define $A\left(z_{0}, R\right)$ to be the set of all finite complex numbers $z$ such that $0<\left|z-z_{0}\right| \leq R$. If $z_{0}$ is infinite, we define $A\left(z_{0}, R\right)$ to be the set of all finite complex numbers $z$ such 
that $1 / R \leq|z|<+\infty$. Note that $A\left(z_{0},+\infty\right)=\Gamma-\left\{z_{0}\right\}$. Also $A\left(z_{0}, R\right)=\Gamma$ if and only if $z_{0}=\infty$ and $R=+\infty$.

With the aid of the above notation we can now state our main result in the following form.

Theorem (1.1). Let $f$ be polyanalytic on $A(\infty, R)$ and represented on $A(\infty, R)$ by means of equation (1.1) where $f_{n}=1$ on $A(\infty, R)$. If $f$ admits the exceptional value zero at the point infinity, then the functions $f_{0}, f_{1}, \cdots, f_{n-1}$ have a nonessential isolated singularity at the point infinity.

First we mention some immediate consequences of Theorem (1.1).

We now need the following result [6].

Lemma (1.2). Let $f$ be polyanalytic on $A(\infty, R)$ and represented on $A(\infty, R)$ by means of equation (1.1) where $f_{n} \not \equiv 0$ on $A(\infty, R)$. If $f$ admits the exceptional value zero at the point infinity, then the function $f_{n}$ also admits the exceptional value zero at the point infinity.

From Theorem (1.1) and Lemma (1.2) we have the following result.

Corollary (1.1). Let $f$ be polyanalytic on $A(\infty, R)$ and represented on $A(\infty, R)$ by means of equation (1.1) where $f_{n} \not \equiv 0$ on $A(\infty, R)$. If $f$ admits the exceptional value zero at the point infinity, then $f_{n}$ admits the exceptional value zero at the point infinity and the functions $f_{0} / f_{n}, f_{1} / f_{n}, \cdots, f_{n-1} / f_{n}$ bave a nonessential isolated singularity at the point infinity.

We next make a preliminary observation. Let $f$ be polyanalytic on $A\left(z_{0}, R\right)$ with an essential isolated singularity at the point $z_{0}$ and suppose that $f$ is represented on $A\left(z_{0}, R\right)$ by means of equation (1.1) where $f_{n} \not \equiv 0$ on $A\left(z_{0}, R\right)$. If the point $z_{0}$ is finite, then $g(z)=f\left(z+z_{0}\right)$ is polyanalytic on $A(0, R)$ with an essential singularity at the point zero. If the point $z_{0}$ is zero, then $g(z)=$ $\bar{z}^{n} f(1 / z)$ is polyanalytic on $A(\infty, R)$ with an essential singularity at the point infinity.

From Corollary (1.1) and the above observation we obtain the following result.

Corollary (1.2). Let $f$ be polyanalytic on $A\left(z_{0}, R\right)$ and represented on $A\left(z_{0}, R\right)$ by means of equation (1.1) and suppose that $f$ admits the exceptional value zero at the point $z_{0^{\circ}}$ If $j=0,1, \cdots, n$ is such that $f_{j} \equiv 0$ on $A\left(z_{0}, R\right)$ then the function $f_{j}$ admits the exceptional value zero at the point $z_{0}$ and for $i=0,1, \cdots, n$ the functions $f_{i} / f_{j}$ bave a nonessential isolated singularity at the point $z_{0}$.

Next from Corollary (1.2) we obtain the following representation for polyanalytic functions which admit an exceptional value at an isolated singularity. 
Corollary (1.3). Let $f$ be polyanalytic on $A\left(z_{0}, R\right)$ and admit the exceptional value zero at the point $z_{0}$. Then there exists a function $h$ analytic and never zero on $A\left(z_{0}, R\right)$ and there exists a function $g$ polyanalytic on $A\left(z_{0}, R\right)$ with a nonessential singularity at the point $z_{0}$ such that $f(z)=h(z) g(z)$, for all $z \in A\left(z_{0}, R\right)$.

It should be noted, in view of Lemmas (1.1) and (1.2), that Theorem (1.1) and Corollaries (1.1), (1.2), and (1.3) are actually equivalent statements.

At this point we give a brief indication of how we propose to establish our main result, Theorem (1.1). In [1], M. B. Balk established his result by applying H. Cartan's theory of meromorphic curves [3] to certain linear combinations of entire functions. If we attempted to establish a proof of Theorem (1.1) by following the procedures utilized by M. B. Balk in [1], we would be dealing with certain linear combinations of functions analytic on an annular neighborhood of a point, for which H. Cartan's theory of meromorphic curves does not apply. However this difficulty can be overcome. In $\$ 2$ we discuss some results concerning functions meromorphic on an annulus. In particular we provide enough details to indicate how the Nevanlinna theory of meromorphic functions can be extended to a similar theory of functions meromorphic on an annulus or, and this is the case of particular interest to us, on an annular neighborhood of a point. On the basis of the results in $\$ 2$, it is easy to see how H. Cartan's theory of meromorphic curves [3] can be extended to an analogous theory concerning linear combinations of functions meromorphic on an annulus or an annular neighborhood of a point. In $\$ 3$ we develop enough of this theory for a certain special case so as to lead to a proof of our main result, Theorem (1.1). Our proof then follows in broad outline the argument utilized by M. B. Balk in [1].

In $\$ 4$ we offer some simple applications of Theorem (1.1). In particular we offer a new proof of the big Picard theorem for polyanalytic functions, a result which was derived by other means in [2].

2. Functions meromorphic on an annulus. In this section we will consider some results concerning functions meromorphic on an annulus. In particular we will briefly indicate how the Nevanlinna theory of meromorphic functions can be extended to a similar theory for functions meromorphic on an annulus and for functions meromorphic on an annular neighborhood of a point.

It will be convenient to introduce the following definitions. First if $f$ is any finite complex valued function which is continuous and never zero on the circumference $|z|=\rho>0$, we define $\Delta_{\rho}$ arg $f$ to be the change in the argument of $f$ along the positively oriented circumference $|z|=\rho$.

We also introduce the so-called $\log$ plus function $\log ^{+} x$ defined for $0 \leq$ $x<+\infty$ by the condition that $\log ^{+} x=0$ if $0 \leq x \leq 1$ and $\log ^{+} x=\log x$ if $1 \leq x<+\infty$. 
Now let $0<R_{0}<R_{1} \leq+\infty$ be fixed and let $/$ be meromorphic on $R_{0} \leq|z|<R_{1}$ such that $f(z) \neq 0, \infty$ for $|z|=R_{0}$. In the discussion to follow we shall frequently be considering integrals of the form

$$
\frac{1}{2 \pi} \int_{0}^{2 \pi} G\left(\left|/\left(R e^{i \theta}\right)\right|\right) d \theta
$$

for $R_{0} \leq R<R_{1}$, where $G(x)$ is some real finite valued function defined and continuous on $0<x<+\infty$. If $R_{0}<R<R_{1}$ is such that $f$ has zeros or poles on $|z|=R$, then the question arises as to whether the integral in (2.1) exists as an improper integral. If we grant that this integral exists for all $R_{0} \leq R<R_{1}$ as a proper or an improper integral, then the further question arises as to whether this integral is a continuous function of $R$ on $R_{0} \leq R<R_{1}$. In this regard, the following observation will prove to be quite useful.

Lemma (2. 1). Suppose there is some positive constant $K$ so that

$$
|G(x)-G(y)|<K|\log x-\log y| \text { for } 0<x, y<+\infty .
$$

Then for each $R_{0}<R<R_{1}$ such that $f$ has zeros or poles on $|z|=R$, the integral in (2.1) exists as an improper integral. Also the integral in (2.1) is a continuous function of $R$ on $R_{0} \leq R<R_{1}$.

We omit the proof of the above result since it is straightforward and involves only routine estimates.

We now need the following result.

Theorem (2.1). Let $0<R_{0}<R_{1} \leq+\infty$ be fixed and let $f$ be meromorphic on $R_{0} \leq|z|<R_{1}$ and suppose fur ther that $f(z) \neq 0, \infty$ for $|z|=R_{0}$. Let $a_{1}, a_{2}, \cdots$ be the zeros and let $b_{1}, b_{2}, \cdots$ be the poles of $f$ on $R_{0}<|z|<R_{1}$, with due regard for multiplicities. Then for all $R_{0} \leq R<R_{1}$ we have that

$$
\begin{aligned}
\frac{1}{2 \pi} \int_{0}^{2 \pi} \log \left|f\left(R e^{i \theta}\right)\right| d \theta= & \sum_{\mu=1}^{\infty} \log ^{+} \frac{R}{\left|a_{\mu}\right|}-\sum_{\mu=1}^{\infty} \log { }^{+} \frac{R}{\left|b_{\mu}\right|} \\
& +\frac{1}{2 \pi} \int_{0}^{2 \pi} \log \left|f\left(R_{0} e^{i \theta}\right)\right| d \theta+\frac{1}{2 \pi} \Delta_{R_{0}} \arg f \log \frac{R}{R_{0}}
\end{aligned}
$$

where the integral on the left-hand side of (2.2) is understood to be improper in case $f$ has zeros or poles on $|z|=R$.

Proof. It suffices to consider the case when $R_{1}$ is finite and $f$ is meromorphic on $R_{0} \leq|z| \leq R_{1}$. Let $a_{1}, a_{2}, \ldots, a_{n}$ be the zeros and let $b_{1}, b_{2}, \cdots, b_{m}$ be the poles of $f$ on $R_{0}<|z| \leq R_{1}$, counting multiplicities. Now the Maclaurin series for $\log (1-z)$ has radius of convergence one, and by Abel's theorem this series is uniformly convergent on every closed subset of the circumference $|z|=1$ 
which does not contain the point one [8, Pp. 134-136]. Hence it is easy to see that

$$
\frac{1}{2 \pi} \int_{0}^{2 \pi} \log \left|1-r e^{i \theta}\right| d \theta=\log ^{+} r
$$

for all $0 \leq r<+\infty$, the above integral being understood to be improper when $r=1$. Consequently,

$$
\frac{1}{2 \pi} \int_{0}^{2 \pi} \log \left|a-b e^{i \theta}\right| d \theta=\log |a|+\log ^{+}|b / a|,
$$

for all finite complex numbers $a$ and $b$ with $a \neq 0$. Now there is a function $g$ meromorphic on $R_{0} \leq|z| \leq R_{1}$ with $g(z) \neq 0, \infty$ for $R_{0} \leq|z| \leq R_{1}$ such that

$$
f(z)=\frac{\Pi_{\mu=1}^{n}\left(z-a_{\mu}\right)}{\Pi_{\mu=1}^{m}\left(z-b_{\mu}\right)} g(z),
$$

for all $R_{0} \leq|z| \leq R_{1}$. From (2.3), (2.4) we deduce that

$$
\begin{aligned}
\frac{1}{2 \pi} \int_{0}^{2 \pi} \log \left|f\left(R e^{i \theta}\right)\right| d \theta-\frac{1}{2 \pi} \int_{0}^{2 \pi} \log \left|f\left(R_{0} e^{i \theta}\right)\right| d \theta \\
=\sum_{\mu=1}^{n} \log ^{+} \frac{R}{\left|a_{\mu}\right|}-\sum_{\mu=1}^{m} \log ^{+} \frac{R}{\left|b_{\mu}\right|}+\frac{1}{2 \pi} \int_{0}^{2 \pi} \log \left|g\left(R e^{i \theta}\right)\right| d \theta \\
-\frac{1}{2 \pi} \int_{0}^{2 \pi} \log \mid g\left(R_{0} e^{i \theta}\right) d \theta,
\end{aligned}
$$

for all $R_{0} \leq R \leq R_{1}$. Now for $R_{0} \leq R \leq R_{1}$, it is easy to see that

$$
R \frac{\partial}{\partial R} \frac{1}{2 \pi} \int_{0}^{2 \pi} \log \left|g\left(R e^{i \theta}\right)\right| d \theta=\frac{1}{2 \pi} \Delta_{R} \arg g=\frac{1}{2 \pi} \Delta_{R_{0}} \arg g=\frac{1}{2 \pi} \Delta_{R_{0}} \arg f .
$$

It therefore follows that

$$
\frac{1}{2 \pi} \int_{0}^{2 \pi} \log \left|g\left(R e^{i \theta}\right)\right| d \theta-\frac{1}{2 \pi} \int_{0}^{2 \pi} \log \left|g\left(R_{0} e^{i \theta}\right)\right| d \theta=\frac{1}{2 \pi} \Delta_{R_{0}} \arg f \log \frac{R}{R_{0}}
$$

for $R_{0} \leq R \leq R_{1}$. From (2.5), (2.6) we obtain (2.2). This proves the result.

The above proof although somewhat long has the merit of being elementary. If we take for granted the fact that the integral on the left-hand side of (2.2) always exists as a proper or an improper integral and that this integral is a continuous function of $R$ on $R_{0} \leq R<R_{1}$, and this can be established directly by elementary estimates or from Lemma (2.1), then a short proof of Theorem (2.1) can be achieved by utilizing Green's theorem as in [7, pp. 164-165].

It should be noted in passing that from (2.2) or (2.5) it follows that the integral on the left-hand side of (2.2) is a continuous function of $R$ on $R_{0} \leq R<R_{1}$. 
The identity in (2.2) is of course an analogue of Jensen's integral formula for functions meromorphic on a disk. The identity in (2.2) will be referred to as Jensen's integral formula for functions meromorphic on an annulus or more simply as Jensen's integral formula for an annulus.

Note that if we let $R_{0}$ approach zero in (2.2) we then obtain Jensen's integral formula for a disk.

Following Nevanlinna we proceed to rewrite Jensen's integral formula for an annulus. From Lemma (2.1) and the inequality $\left|\log ^{+} x-\log ^{+} y\right| \leq|\log x-\log y|$ which is valid for $0<x, y<+\infty$ we see that the integral $1 / 2 \pi^{-1} \int_{0}^{2 \pi} \log ^{+}\left|f\left(R e^{i \theta}\right)\right| d \theta$ exists as an improper integral in case $f$ has poles on $|z|=R$ and we also see that this integral is a continuous function of $R$ on $R_{0} \leq R<R_{1}$. From the identity $\log x=\log ^{+} x-\log ^{+}(1 / x)$, we see that (2.2) can be written in the more symmetrical form

$$
\begin{aligned}
\frac{1}{2 \pi} \int_{0}^{2 \pi} \log ^{+}\left|f\left(R e^{i \theta}\right)\right| d \theta+\sum_{\mu=1}^{\infty} \log ^{+} \frac{R}{\left|b_{\mu}\right|} \\
=\frac{1}{2 \pi} \int_{0}^{2 \pi} \log ^{+}\left|\frac{1}{f\left(R e^{i \theta}\right)}\right| d \theta+\sum_{\mu=1}^{\infty} \log ^{+} \frac{R}{\left|a_{\mu}\right|} \\
+\frac{1}{2 \pi} \int_{0}^{2 \pi} \log \left|f\left(R_{0} e^{i \theta}\right)\right| d \theta+\frac{1}{2 \pi} \Delta_{R_{0}} \arg f \log \frac{R}{R_{0}},
\end{aligned}
$$

for $R_{0} \leq R<R_{1}$.

In order to make the symmetry of (2.7) more evident still we make the following definitions. Assume the hypothesis of Theorem (2.1). Then for $R_{0} \leq R<$ $R_{1}$, we let

$$
\begin{gathered}
m(R, f)=m(R, f, \infty)=\frac{1}{2 \pi} \int_{0}^{2 \pi} \log { }^{+}\left|f\left(R e^{i \theta}\right)\right| d \theta \\
m(R, 1 / f)=m(R, f, 0)=\frac{1}{2 \pi} \int_{0}^{2 \pi} \log ^{+}\left|\frac{1}{f\left(R e^{i \theta}\right)}\right| d \theta
\end{gathered}
$$

The expression $m(R, f)$ is called the proximity function of $f$ and is evidently a continuous function of $R$ on $R_{0} \leq R<R_{1}$. Also for $R_{0} \leq R<R_{1}$ we define $N\left(R, R_{0}, f\right)=N(R, f)=N\left(R, R_{0}, f, \infty\right)=N(R, f, \infty)$ and $N\left(R, R_{0}, 1 / f\right)=$ $N(R, 1 / f)=N\left(R, R_{0}, f, 0\right)=N(R, f, 0)$ by the condition that

$$
N(R, f)=\sum_{\mu=1}^{\infty} \log ^{+} \frac{R}{\left|b_{\mu}\right|}, \quad N(R, 1 / f)=\sum_{\mu=1}^{\infty} \log ^{+} \frac{R}{\left|a_{\mu}\right|} .
$$


The expression $N(R, f)$ is called the counting function for $f$. Also for $R_{0} \leq R<R_{1}$, we let $n\left(R, R_{0}, f\right)=n(R, f)=n\left(R, R_{0}, f, \infty\right)=n(R, f, \infty)$ denote the number of poles of $f$ in $R_{0}<|z|<R$, counting multiplicities, and we let $n\left(R, R_{0}, 1 / f\right)=n(R, 1 / f)=n\left(R, R_{0}, f, 0\right)=n(R, f, 0)$ denote the number of zeros of $f$ in $R_{0}<|z|<R$, counting multiplicities. Note that

$$
N(R, f)=\int_{R_{0}}^{R} \frac{n(t, f)}{t} d t, \quad N(R, 1 / f)=\int_{R_{0}}^{R} \frac{n(t, 1 / f)}{t} d t,
$$

for $R_{0} \leq R<R_{1}$.

From the above definitions we see that (2.7) can be written as

$$
m(R, f)+N(R, f)=m(R, 1 / f)+N(R, 1 / f)
$$

$$
+\frac{1}{2 \pi} \int_{0}^{2 \pi} \log \left|f\left(R_{0} e^{i \theta}\right)\right| d \theta+\frac{1}{2 \pi} \Delta_{R_{0}} \arg f \log \frac{R}{R_{0}}
$$

for $R_{0} \leq R<R$.

Again, assuming the hypothesis of Theorem (2.1), we define $T\left(R, R_{0}, f\right)=$ $T(R, f)$ by the condition that

$$
T(R, f)=m(R, f)+N(R, f)
$$

for $R_{0} \leq R<R_{1}$. The expression $T(R, f)$ is called the characteristic function of $f$ and is evidently a continuous function of $R$ on $R_{0} \leq R<R_{1}$.

From (2.11), (2.12) we obtain the following formulation of Jensen's integral formula for an annulus.

Theorem (2.2). Assume the hypothesis of Theorem (2.1). Then for $R_{0} \leq R<R_{1}$ we have that

$$
T(R, f)=T(R, 1 / f)+\frac{1}{2 \pi} \int_{0}^{2 \pi} \log \left|f\left(R_{0} e^{i \theta}\right)\right| d \theta+\frac{1}{2 \pi} \Delta_{R_{0}} \arg f \log \frac{R}{R_{0}}
$$

Note that if we let $R_{0}$ approach zero in (2.13), we obtain Nevanlinna's form of Jensen's integral formula for functions meromorphic on a disk.

From the inequalities

$$
\log ^{+}\left|\prod_{\mu=1}^{p} a_{\mu}\right| \leq \sum_{\mu=1}^{p} \log ^{+}\left|a_{\mu}\right|, \quad \log ^{+}\left|\sum_{\mu=1}^{p} a_{\mu}\right| \leq \sum_{\mu=1}^{p} \log ^{+}\left|a_{\mu}\right|+\log p,
$$

we easily deduce that

$$
m\left(R, \prod_{\mu=1}^{p} f_{\mu}\right) \leq \sum_{\mu=1}^{p} m\left(R, f_{\mu}\right), \quad m\left(R, \sum_{\mu=1}^{p} f_{\mu}\right) \leq \sum_{\mu=1}^{p} m\left(R, f_{\mu}\right)+\log p .
$$


Also

$$
N\left(R, \prod_{\mu=1}^{p} f_{\mu}\right) \leq \sum_{\mu=1}^{p} N\left(R, f_{\mu}\right), \quad N\left(R, \sum_{\mu=1}^{p} f_{\mu}\right) \leq \sum_{\mu=1}^{p} N\left(R, f_{\mu}\right) .
$$

It therefore follows that

$$
T\left(R, \prod_{\mu=1}^{p} f_{\mu}\right) \leq \sum_{\mu=1}^{p} T\left(R, f_{\mu}\right), \quad T\left(R, \sum_{\mu=1}^{p} f_{\mu}\right) \leq \sum_{\mu=1}^{p} T\left(R, f_{\mu}\right)+\log p
$$

In particular we see that

$$
|T(R, f)-T(R, f-a)| \leq \log ^{+}|a|+\log 2,
$$

for any finite complex number $a$.

Now from Theorem (2.2) and the above inequality we deduce the first fundamental theorem for functions meromorphic on an annulus.

Theorem (23). Assume the hypothesis of Theorem (2.1). Let a be any finite complex number such that $f(z) \neq a$ for $|z|=R_{0}$. Then for all $R_{0} \leq R<R_{1}$ we have that

$$
\begin{aligned}
T\left(R, \frac{1}{f-a}\right)= & T(R, f)-\frac{1}{2 \pi} \int_{0}^{2 \pi} \log \left|f\left(R_{0} e\right)^{i \theta}-a\right| d \theta \\
& -\frac{1}{2 \pi} \Delta_{R_{0}} \arg (f-a) \log \frac{R}{R_{0}}+\epsilon(a, R, f),
\end{aligned}
$$

where $|\epsilon(a, R, f)| \leq \log ^{+}|a|+\log 2$.

As a simple consequence of Theorem (2.3) we mention the following result which we shall utilize later.

Corollary (2.1). Let $f$ be meromorphic on $0<R_{0} \leq|z|<+\infty$. Suppose there is an increasing sequence $\left\{R_{j}\right\}, j=1,2, \cdots$, of real numbers greater than $R_{0}$ and diverging to $+\infty$ so that $T\left(R_{j}, f\right)<A \log R_{j}$, for $j=1,2, \cdots$, where $A$ is some positive constant. Then $f$ has only a finite number of poles on $R_{0} \leq|z|<$ $+\infty$ and $f$ has a nonessential isolated singularity at the point infinity.

Again let $0<R_{0}<R_{1} \leq+\infty$ to be fixed and let $f$ be a function meromorphic on $R_{0} \leq|z|<+\infty$ with $f(z) \neq 0, \infty$ for $|z|=R_{0}$. Our next goal in this section is to obtain a suitable estimate for $m\left(R, f^{\prime} / f\right)$ in terms of $\log ^{+} T(R, f)$. We shall consider only the case when $R_{1}=+\infty$. To achieve this goal we shall use a method similiar to the differential geometric method utilized in [7, pp. 258-260].

Now let $g(x)$ be a real finite valued function defined and continuous on $0 \leq x \leq+\infty$ and suppose that $g^{\prime}(x)$ exists and is continuous on $0<x<+\infty$. We 
now define $G(x)$ for $0<x<+\infty$ by the condition that $G^{\prime}(x)=g(x) / x$.

We now need the following result.

Lemma (2.2). Let $D$ be a domain in $\Gamma$ which is bounded by a finite system of closed analytic curves $\gamma$. Next suppose that $f$ is a function meromorphic on $D+$ $\gamma$ such that $f(z) \neq 0, \infty$ for $z$ on $\gamma$. Let $n$ and $m$ denote the number of zeros and poles, respectively, of $f$ in $D$, counting multiplicities. Then

$$
\frac{1}{2 \pi} \int_{\gamma} \frac{\partial G(|f|)}{\partial n} d s=\frac{1}{2 \pi} \iint_{D} g^{\prime}(|f|)|f|^{-1}\left|f^{\prime}\right|^{2}+n g(0)-m g(\infty),
$$

where we integrate along $\gamma$ in such a manner that the domain $D$ remains at the left as we traverse $\gamma$, where $s$ denotes arc length along $\gamma$, and where $\partial / \partial n$ denotes differentiation along the normal to $y$ out of $D$.

Proof. 'Let $z_{1}, z_{2}, \cdots, z_{p}$ be the distinct points of $D$ at which $f$ has a zero or a pole. For $k=1,2, \cdots, p$ let $z_{k}$ be a zero of $f$ of multiplicity $m_{k} \geq 1$ or a pole of $f$ of multiplicity $\left(-m_{k}\right) \geq 1$. There is some $\sigma_{0}>0$ so that for $0<\sigma<\sigma_{0}$ and for $k=1,2, \ldots, p$ the disks $D_{k}=D\left(z_{k}, \sigma\right)$ with center $z_{k}$ and radius $\sigma$ are disjoint and contained in $D$. For $k=1,2, \cdots, p$ let $\gamma_{k}=\gamma\left(z_{k}, \sigma\right)$ be the boundary of $D\left(z_{k}, \sigma\right)$

oriented in the positive sense. For $0<\sigma<\sigma_{0}$, let $D_{\sigma}=D-\Sigma D_{k}, k=1,2, \cdots, p$. Hence the boundary $\gamma_{\sigma}$ of $D_{\sigma}$ is given by $\gamma_{\sigma}=\gamma+\Sigma \gamma_{k}, k=1,2, \cdots, p$. From Green's theorem we see that

$$
\frac{1}{2 \pi} \int_{\gamma_{\sigma}} \frac{\partial G(|f|)}{\partial n} d s=\frac{1}{2 \pi} \iint_{D_{\sigma}} \Delta G(|f|),
$$

for $0<\sigma<\sigma_{0}$, where $\Delta=\partial^{2} / \partial x^{2}+\partial^{2} / \partial y^{2}$ is the Laplacian. It is easy to verify that $\Delta G(|f|)=g^{\prime}(|f|)|f|^{-1}\left|f^{\prime}\right|^{2}$. Consequently,

$$
\frac{1}{2 \pi} \int_{\gamma} \frac{\partial G(|f|)}{\partial n} d s-\sum_{k=1}^{p} \frac{1}{2 \pi} \int_{\gamma_{k}} \frac{\partial G(|f|)}{\partial n} d s=\frac{1}{2 \pi} \iint_{D \sigma} g^{\prime}(|f|)|f|^{-1}\left|f^{\prime}\right|^{2}
$$

for $0<\sigma<\sigma_{0^{\circ}}$. On the other hand, for $0<\sigma<\sigma_{0}$ and for $k=1,2, \cdots, p$ we see that

$$
\frac{1}{2 \pi} \int_{\gamma_{k}} \frac{\partial G(|f|)}{\partial n} d s=\frac{1}{2 \pi} \int_{0}^{2 \pi} g\left(\left|f\left(w_{k}\right)\right|\right) R\left(w_{k} \frac{f^{\prime}\left(w_{k}\right)}{f\left(w_{k}\right)}\right) d \theta
$$

where $w_{k}=z_{. k}+\sigma e^{i \theta}$. From the above identity we deduce that

$$
\lim _{\sigma \rightarrow 0} \frac{1}{2 \pi} \int_{\gamma_{k}} \frac{\partial G(|f|)}{\partial n} d s=m_{k} g\left(\left|f\left(z_{k}\right)\right|\right)
$$


for $k=1,2, \cdots, p$. From the above result and from (2.20) we obtain (2.19). This establishes the result.

Note that the double integral in (2.19) is understood to be improper in the sense indicated in the above proof.

For identities quite similar to the identity in (2.19) see [4] and [5, p. 10].

The identity in (2.19) leads to a variety of interesting and useful formulas concerning meromorphic functions by suitably choosing $D$ and $g(x)$. We are particularly interested in the case where $D$ is an annulus.

Now let $0<R_{n}<R_{1} \leq+\infty$ be fixed and let. $f$ be meromorphic on $R_{0} \leq|z|<R_{1}$ such that $f(z) \neq 0, \infty$ for $|z|=R_{n}$. As an immediate consequence of Lemma (2.2) we have the following result.

Corollary (2.2). For all $R_{0} \leq R<R_{1}$ such that $f(z) \neq 0, \infty$ for $|z|=R$ we have that

$$
\begin{aligned}
\frac{1}{2 \pi} \int_{0}^{2 \pi} R & \frac{\partial G\left(\left|f\left(R e^{i \theta}\right)\right|\right)}{\partial R} d \theta \\
= & \frac{1}{2 \pi} \int_{R_{0}}^{R} \int_{0}^{2 \pi} g^{\prime}\left(\left|f\left(r e^{i \theta}\right)\right|\right)\left|f\left(r e^{i \theta}\right)\right|^{-1}\left|f^{\prime}\left(r e^{i \theta}\right)\right|^{2} r d r d \theta \\
& +g(0) n(R, f, 0)-g(\infty) n(R, f, \infty) \\
& +\frac{1}{2 \pi} \int_{0}^{2 \pi} g\left(\left|f\left(R_{0} e^{i \theta}\right)\right|\right) \Re\left(R_{0} e^{i \theta} \frac{f^{\prime}\left(R_{0} e^{i \theta}\right)}{f\left(R_{0} e^{i \theta}\right)}\right) d \theta .
\end{aligned}
$$

Before we can utilize the identity in (2.21) we need some preliminary observations which we state as lemmas.

Lemma (2.3). If $R_{0} \leq R<R_{1}$ is such that $f$ has zeros or poles on $|z|=R$, then the integral in (2.1) exists as an improper integral. Moreover this integral is a continuous function of $R$ on $R_{0} \leq R<R_{1}$.

Proof. There is some positive constant $K$ so that $|g(x)| \leq K$ for all $0 \leq$ $x \leq+\infty$. Now for $0<x, y<+\infty$ we have that $G(x)-G(y)=\int_{y}^{x}(g(t) / t) d t$. Hence we see that $|G(x)-G(y)| \leq K|\log x-\log y|$ for $0<x, y<+\infty$. The result now follows from Lemma (2.1).

We also need the following result.

Lemma (2.4). Suppose that $g^{\prime}(x) \geq 0$ for all $0<x<+\infty$. Then the double integral in (2.21) is a continuous function of $R$ on $R_{0} \leq R<R_{1}$.

Proof. Denote this double integral by $V(R)$ and let $I$ denote the set of all $R_{0} \leq R<R_{1}$ such that $f(z) \neq 0, \infty$ for $|z|=R$. Evidently $V(R)$ is well defined, continuous, and increasing on $I$. Let $R_{0}<R_{2}<R_{1}$ be such that $f$ has zeros or 
poles on $|z|=R_{2}$. Now in order to show that $V(R)$ may be defined at $R=R_{2}$ in such a way that $V(R)$ is continuous at $R=R_{2}$ it suffices to show that if $R_{0}<R<R_{2}<S<R_{1}$ then $V(S)-V(R) \rightarrow 0$ as $R, S \rightarrow R_{2}$. In order to establish this fact we proceed as follows. First there is some $0<\sigma_{0}<R_{1}-R_{2}, R_{2}-R_{0}$ so that the only zeros or poles of $f$ on $R_{2}-\sigma_{0} \leq|z| \leq R_{2}+\sigma_{0}$ are on the circumference $|z|=R_{2}$. Let $z_{1}, z_{2}, \cdots, z_{p}$ be the distinct points on $|z|=R_{2}$ at which $f$ has a zero or a pole. For $k=1,2, \cdots, p$ let $z_{k}$ be a zero of $f$ of multiplicity $m_{k} \geq 1$ or a pole of $f$ of multiplicity $\left(-m_{k}\right) \geq 1$. Next there is some $0<$ $\sigma_{1}<\sigma_{0}$ so that for $0<\sigma<\sigma_{1}$ and for $k=1,2, \cdots, p$ the disks $D_{k}=D\left(z_{k}, \sigma\right)$ with center $z_{k}$ and radius $\sigma$, which are contained in the annulus $R_{2}-\sigma_{0} \leq$ $|z| \leq R_{2}+\sigma_{0}$, are disjoint. For $k=1,2, \cdots, p$ let $\gamma_{k}=\gamma\left(z_{k}, \sigma\right)$ denote the boundary of $D_{k}=D\left(z_{k}, \sigma\right)$ oriented in the positive sense. From Lemma (2.2) we see that for $0<\sigma<\sigma_{1}$ and $k=1,2, \cdots, p$ that

$$
\begin{aligned}
\frac{1}{2 \pi} \int_{D_{k}} g^{\prime}(|f|)|f|^{-1}\left|f^{\prime}\right|^{2} & =\frac{1}{2 \pi} \int_{\gamma_{k}} \frac{\partial G(|f|)}{\partial n} d s-m_{k} g\left(\left|f\left(z_{k}\right)\right|\right) \\
& =\frac{1}{2 \pi} \int_{0}^{2 \pi} g\left(\left|f\left(w_{k}\right)\right|\right) R\left(w_{k} \frac{f^{\prime}\left(w_{k}\right)}{f\left(w_{k}\right)}\right) d \theta-m_{k} g\left(\left|f\left(z_{k}\right)\right|\right),
\end{aligned}
$$

where $w_{k}=z_{k}+\sigma e^{i \theta}$. For $0<\sigma<\sigma_{1}$ and $k=1,2, \cdots, p$ let $\epsilon\left(z_{k}, \sigma\right)$ denote the double integral on $D_{k}$ in the above equation. Clearly $\epsilon\left(z_{k}, \sigma\right) \rightarrow 0$ as $\sigma \rightarrow 0$ for $k=1,2, \cdots, p$. Let $\epsilon>0$ be given. There is some fixed $0<\sigma_{2}<\sigma_{1}$ so that $\epsilon\left(z_{k}, \sigma_{2}\right)<\epsilon / 2 p$ for $k=1,2, \cdots, p$. There is some positive constant $M$ so that $g^{\prime}(|f(z)|)|f(z)|^{-1}\left|f^{\prime}(z)\right|^{2}<M$ for all $R_{2}-\sigma_{0} \leq|z| \leq R_{2}+\sigma_{0}$ such that $z \notin$ $D\left(z_{k}, \sigma_{2}\right)$ for $k=1,2, \cdots, p$. Thus if $R_{2}-\sigma_{0}<R<R_{2}<S<R_{2}+\sigma_{0}$ we deduce that $0 \leq V(S)-V(R)<\epsilon / 2+\left(S^{2}-R^{2}\right) M / 2$. The result now follows from this estimate.

We are now interested in using the identity in (2.21) when $G(x)=\left(1+\log ^{2} x\right)^{1 / 2}$ so that $g(x)=\log x /\left(1+\log ^{2} x\right)^{1 / 2}$ and $g^{\prime}(x)=x /\left(1+\log ^{2} x\right)^{3 / 2}$.

Now let $0<R_{0}<+\infty$ be fixed and let $/$ be meromorphic on $R_{0} \leq|z|<+\infty$ with $f(z) \neq 0, \infty$ for $|z|=R_{0}$. For $R \geq R_{0}$ we define $U(R)=U(R, f)$ by the condition that

$$
U(R)=\frac{1}{2 \pi} \int_{0}^{2 \pi}\left(1+\log ^{2}\left|f\left(R e^{i \theta}\right)\right|\right)^{1 / 2} d \theta+N\left(R, R_{0}, f, 0\right)
$$

$$
\begin{aligned}
& -N\left(R, R_{0}, f, \infty\right)-\frac{1}{2 \pi} \int_{0}^{2 \pi}\left(1+\log ^{2}\left|f\left(R_{0} e^{i \theta}\right)\right|\right)^{1 / 2} d \theta \\
& -\log \frac{R}{R_{0}} \frac{1}{2 \pi} \int_{0}^{2 \pi} \frac{\log \left|f\left(R_{0} e^{i \theta}\right)\right|}{\left(1+\log ^{2}\left|f\left(R_{0} e^{i \theta}\right)\right|\right)^{1 / 2}} R\left(R_{0} e^{i \theta} \frac{f^{\prime}\left(R_{0} e^{i \theta}\right)}{f\left(R_{0} e^{i \theta}\right)}\right) d \theta .
\end{aligned}
$$


In view of Lemma (2.3), the function $U(R)$ is well defined and continuous on $R \geq R_{0}$. Now let $t=\log R$ so that $d / d t=R d / d R$. From Corollary (2.2) we see that if $R \geq R_{0}$ is such that $f(z) \neq 0, \infty$ for $|z|=R$, then

$$
\frac{d U(R)}{d t}=\frac{1}{2 \pi} \int_{R_{0}}^{R} \int_{0}^{2 \pi}\left|\frac{f^{\prime}\left(r e^{i \theta}\right)}{f\left(r e^{i \theta}\right)}\right|^{2} \frac{r d r d \theta}{\left(1+\log ^{2}\left|f\left(r e^{i \theta}\right)\right|\right)^{3 / 2}} \cdot
$$

From Lemma (2.4) we see that $d U(R) / d t$ is continuous on $R \geq R_{0}$. Further for all $R \geq R_{0}$ such that $f(z) \neq 0, \infty$ for $|z|=R$ we see that

$$
\frac{d^{2} U(R)}{d t^{2}}=\frac{R^{2}}{2 \pi} \int_{0}^{2 \pi}\left|\frac{f^{\prime}\left(R e^{i \theta}\right)}{f\left(\operatorname{Re}^{i \theta}\right)}\right|^{2} \frac{d \theta}{\left(1+\log ^{2}\left|f\left(\operatorname{Re}^{i \theta}\right)\right|\right)^{3 / 2}}
$$

We note that $d^{2} U(R) / d t^{2}$ exists and is continuous on $R \geq R_{0}$ except possibly for an isolated set of points.

We now wish to obtain an upper estimate for $U(R)$ in terms of $T(R, f)$. From (2.8), (2.12), (2.13), (2.22) and the inequality $\left(1+\log ^{2} x\right)^{1 / 2} \leq 1+\log ^{+} x+$ $\log ^{+}(1 / x)$ which is valid for $0<x<+\infty$ we see that

$$
\begin{aligned}
U(R) \leq & 1+2 T(R, f)-\frac{1}{2 \pi} \Delta_{R_{0}} \arg f \log \frac{R}{R_{0}} \\
& -\log \frac{R}{R_{0}} \frac{1}{2 \pi} \int_{0}^{2 \pi} \frac{\log \left|\left(R_{0} e^{i \theta}\right)\right|}{\left(1+\log \left|f\left(R_{0} e^{i \theta}\right)\right|\right)^{1 / 2}} R\left(R_{0} e^{i \theta} \frac{f^{\prime}\left(R_{0} e^{i \theta}\right)}{f\left(R_{0} e^{i \theta}\right)}\right) d \theta \\
& -\frac{1}{2 \pi} \int_{0}^{2 \pi} \log \left|f\left(R_{0} e^{i \theta}\right)\right| d \theta-\frac{1}{2 \pi} \int_{0}^{2 \pi}\left(1+\log ^{2}\left|f\left(R_{0} e^{i \theta}\right)\right|\right)^{1 / 2} d \theta \\
& \leq 1+2 T(R, f)-\log \frac{R}{R_{0}} \frac{1}{2 \pi} \int_{0}^{2 \pi}\left(\frac{\log \left|f\left(R_{0} e^{i \theta}\right)\right|}{\left.\left(1+\log ^{2}\left|f\left(R_{0} e^{i \theta}\right)\right|\right)^{1 / 2}+1\right)} \cdot R\left(R_{0} e^{i \theta} \frac{f^{\prime}\left(R_{0} e^{i \theta}\right)}{f\left(R_{0} e^{i \theta}\right)}\right) d \theta\right. \\
& \leq 1+2 T(R, f)+2 R_{0} \log \frac{R}{R_{0}} \frac{1}{2 \pi} \int_{0}^{2 \pi}\left|\frac{f^{\prime}\left(R_{0} e^{i \theta}\right)}{f\left(R_{0} e^{i \theta}\right)}\right| d \theta \\
& \leq 1+2 T(R, f)+2 R \frac{1}{2 \pi} \int_{0}^{2 \pi}\left|\frac{f^{\prime}\left(R_{0} e^{i \theta}\right)}{f\left(R_{0} e^{i \theta}\right)}\right| d \theta,
\end{aligned}
$$

for all $R \geq R_{0}$.

Hence we see that 


$$
\log ^{+} U(R) \leq \log { }^{+} T(R, f)+\log { }^{+} R+\frac{1}{2 \pi} \int_{0}^{2 \pi}\left|\frac{f^{\prime}\left(R_{0} e^{i \theta}\right)}{f\left(R_{0} e^{i \theta}\right)}\right| d \theta+4 \log 2
$$

for all $R \geq R_{0}$.

We next wish to obtain a suitable upper estimate for $m\left(R, f^{\prime} / f\right)$ in terms of $d^{2} U(R) / d t^{2}$. To do this it will be convenient to introduce the plus function $x^{+}$ defined for $0 \leq x<+\infty$ by the condition that $x^{+}=1$ if $0 \leq x \leq 1$ and $x^{+}=x$ if $1 \leq x<+\infty$. Equivalently we could have defined $x^{+}$by the condition that $x^{+}=$ $e^{\log ^{+} x}$ for $0 \leq x<+\infty$. The plus function $x^{+}$enjoys many pleasant properties just as the $\log$ plus function $\log ^{+} x$ does. From (2.24) and the inequality $x \leq x^{+} \leq$ $x+1$ which is valid for $0 \leq x<+\infty$ we see that

$$
\frac{d^{2} U(R)}{d t^{2}} \geq \frac{R^{2}}{2 \pi} \int_{0}^{2 \pi} \frac{\left(\left|f^{\prime}\left(R e^{i \theta}\right) / f\left(R e^{i \theta}\right)\right|^{2}\right)^{+}-1}{\left(1+\log ^{2}\left|f\left(R e^{i \theta}\right)\right|\right)^{3 / 2}} d \theta
$$

for all $R \geq R_{0}$ such that $f(z) \neq 0, \infty$ for $|z|=R$. Hence we see that

$$
1+\frac{1}{R^{2}} \frac{d^{2} U(R)}{d t^{2}} \geq \frac{1}{2 \pi} \int_{0}^{2 \pi}\left(\left|\frac{f^{\prime}\left(R e^{i \theta}\right)}{f\left(R e^{i \theta}\right)}\right|^{2}\right)^{+} \frac{d \theta}{\left(1+\log ^{2}\left|f\left(R e^{i \theta}\right)\right|\right)^{3 / 2}},
$$

for all $R \geq R_{0}$ such that $f(z) \neq 0, \infty$ so for $|z|=R$. If we now take the logarithm of both sides of the above inequality and if we apply the theorem on the arithmetic and geometric mean $[7$, p. 251] we deduce that

$$
\begin{aligned}
\log \left(1+\frac{1}{R^{2}} \frac{d^{2} U(R)}{d t^{2}}\right) \geq & \frac{1}{2 \pi} \int_{0}^{2 \pi} \log \left(\left|\frac{f^{\prime}\left(R e^{i \theta}\right)}{f\left(R e^{i \theta}\right)}\right|^{2}\right)+ \\
& -\frac{1}{2 \pi} \int_{0}^{2 \pi} \log \left(1+\log ^{2}\left|f\left(R e^{i \theta}\right)\right|\right)^{3 / 2} d \theta
\end{aligned}
$$

for all $R \geq R_{0}$ such that $f(z) \neq 0, \infty$ for $|z|=R$. Now from the above estimate and from (2.8) and from the identities $\log x^{+}=\log ^{+} x$ and $\log ^{+} x^{2}=2 \log ^{+} x$ which are valid for $0 \leq x<+\infty$ we deduce that

$$
2 m\left(R, f^{\prime} / f\right) \leq \log \left(1+\frac{1}{R^{2}} \frac{d^{2} U(R)}{d t^{2}}\right)+\frac{3}{2 \pi} \int_{0}^{2 \pi} \log \left(1+\log ^{2}\left|f\left(R e^{i \theta}\right)\right|\right)^{1 / 2} d \theta
$$

for all $R \geq R_{0}$ such that $f(z) \neq 0, \infty$ for $|z|=R$. If we again apply the theorem on the arithmetic and geometric mean $[7$, p. 251] we see that

$$
\begin{aligned}
\frac{1}{2 \pi} \int_{0}^{2 \pi} & \log \left(1+\log ^{2}\left|f\left(R e^{i \theta}\right)\right|\right)^{1 / 2} d \theta \leq \log \frac{1}{2 \pi} \int_{0}^{2 \pi}\left(1+\log ^{2}\left|f\left(R e^{i \theta}\right)\right|\right)^{1 / 2} d \theta \\
& \leq \log \frac{1}{2 \pi} \int_{0}^{2 \pi}\left(1+\log ^{+}\left|f\left(R e^{i \theta}\right)\right|+\log ^{+} \frac{1}{\left|f\left(R e^{i \theta}\right)\right|}\right) d \theta \\
& =\log ^{+}(1+m(R, f)+m(R, 1 / f))
\end{aligned}
$$


for all $R \geq R_{0}$. From the above estimate and from (2.12), (2.13) we see that

$$
\begin{aligned}
\frac{1}{2 \pi} \int_{0}^{2 \pi} & \log \left(1+\log ^{2}\left|f\left(R e^{i \theta}\right)\right|\right)^{1 / 2} d \theta \\
& \leq \log ^{+}\left(1+2 T(R, f)-\frac{1}{2 \pi} \int_{0}^{2 \pi} \log \left|f\left(R_{0} e^{i \theta}\right)\right| d \theta-\log \frac{R}{R_{0}} \frac{1}{2 \pi} \Delta_{R_{0}} \arg f\right) \\
& \leq \log ^{+}\left(2 T(R, f)+\frac{1}{2 \pi} \int_{0}^{2 \pi} \frac{1}{\left|f\left(R_{0} e^{i \theta}\right)\right|} d \theta+R \frac{1}{2 \pi} \int_{0}^{2 \pi}\left|\frac{f^{\prime}\left(R_{0} e^{i \theta}\right) \mid}{f\left(R_{0} e^{i \theta}\right)}\right| d \theta\right) \\
& \leq \log ^{+}\left(2 T(R, f)+R^{+} \frac{1}{2 \pi} \int_{0}^{2 \pi} \frac{\left|f^{\prime}\left(R_{0} e^{i \theta}\right)\right|+1}{\left|f\left(R_{0} e^{i \theta}\right)\right|} d \theta\right),
\end{aligned}
$$

for all $R \geq R_{0}$. Hence we obtain that

$$
\begin{aligned}
\frac{1}{2 \pi} \int_{0}^{2 \pi} \log (1 & \left.+\log ^{2}\left|f\left(R e^{i \theta}\right)\right|\right)^{1 / 2} d \theta \\
\leq & \log ^{+} T(R, f)+\log ^{+} R+\frac{1}{2 \pi} \int_{0}^{2 \pi} \frac{\left|f^{\prime}\left(R_{0} e^{i \theta}\right)\right|+1}{\left|f\left(R_{0} e^{i \theta}\right)\right|} d \theta+2 \log 2,
\end{aligned}
$$

for all $R \geq R_{0}$. Now from the above estimate and from (2.26) we deduce that

$$
\begin{aligned}
2 m\left(R, f^{\prime} / f\right) \leq & \log \left(1+\frac{1}{R^{2}} \frac{d^{2} U(R)}{d t^{2}}\right)+3 \log ^{+} T(R, f) \\
& +\frac{3}{2 \pi} \int_{0}^{2 \pi} \frac{\left|f^{\prime}\left(R_{0} e^{i \theta}\right)\right|+1}{\left|f\left(R_{0} e^{i \theta}\right)\right|} d \theta+6 \log 2,
\end{aligned}
$$

for all $R \geq R_{0}$ such that $f(z) \neq 0, \infty$ for $|z|=R$.

Now in order to obtain a suitable upper estimate for $m\left(R, f^{\prime} / f\right)$ in terms of $\log ^{+} T(R, f)$ by means of (2.25), (2.27) we need to obtain an appropriate upper estimate for $d^{2} U(R) / d t^{2}$ in terms of $U(R)$. For this purpose the following lemma will suffice.

Lemma (2.5). Let $f(x)$ be continuous and increasing for $0 \leq x<+\infty$ with $f(0)=0$. Also let $f^{\prime}(x)$ exist and be continuous on $0 \leq x<+\infty$ except possibly for an isolated set of points. If $a>0$ then $f^{\prime}(x)<(f(x)+a)^{2}$ for all $0 \leq x<+\infty$ except possibly for a set of measure at most $1 / a$.

For a proof of a more general result see [7, p. 253].

If $a>0$ a double application of the above lemma leads immediately to the estimate $d^{2} U(R) / d t^{2}<R\left|R(U(R)+2 / a)^{2}+2 / a\right|^{2}$ which is valid for all $R \geq R_{0}$ except possibly for a set of measure at most $a$. From the above estimate and from (2.14) we see that if $a>0$ then 


$$
\log \left(1+\frac{1}{R^{2}} \frac{d^{2} U(R)}{d t^{2}}\right) \leq 4 \log ^{+} U(R)+2 \log ^{+} R+\log ^{+} \frac{1}{R}+6 \log ^{+} \frac{1}{a}+11 \log 2,
$$

for all $R \geq R_{0}$ except possibly for a set of measure at most $a$. Now from the above estimate and from (2.25) we see that, for all $a>0$,

$$
\begin{aligned}
\log \left(1+\frac{1}{R^{2}} \frac{d^{2} U(R)}{d t^{2}}\right) \leq & 4 \log ^{+} T(R, f)+6 \log ^{+} R+\log ^{+} \frac{1}{R} \\
& +4 \frac{1}{2 \pi} \int_{0}^{2 \pi}\left|\frac{f^{\prime}\left(R_{0} e^{i \theta}\right)}{f\left(R_{0} e^{i \theta}\right)}\right| d \theta+6 \log ^{+} \frac{1}{a}+27 \log 2
\end{aligned}
$$

for all $R \geq R_{0}$ except possibly for a set of measure at most $a$. Now from the above estimate and from (2.27) we obtain the following result.

Theorem (2.4). Let $f$ be meromorphic on $0<R_{0} \leq|z|<+\infty$ such that $f(z) \neq 0, \infty$ for $|z|=R_{0}$. Then for all $a>0$ we have that

$$
\begin{aligned}
m\left(R, f^{\prime} / f\right) \leq & 4 \log ^{+} T(R, f)+3 \log ^{+} R+\log ^{+} \frac{1}{R} \\
& +4 \frac{1}{2 \pi} \int_{0}^{2 \pi} \frac{\left|f^{\prime}\left(R_{0} e^{i \theta}\right)\right|+1}{\left|f\left(R_{0} e^{i \theta}\right)\right|} d \theta+3 \log ^{+} \frac{1}{a}+17 \log 2,
\end{aligned}
$$

for all $R \geq R_{0}$ except possibly for a set $I=I(a, f)$ of measure at most $a$.

It should be noted on the basis of the above result that Nevanlinna's second fundamental in the theory of meromorphic functions can be readily extended to functions meromorphic on $0<R_{0} \leq|z|<+\infty$ by arguing no differently than in [7, pp. 31-34]. Note further that by virtue of the above result that H. Cartan's theory of meromorphic curves can be easily extended to an analogous theory of meromorphic curves on $0<R_{0} \leq|z|<+\infty$ by reasoning similar to that utilized by H. Cartan in [3].

3. Proof of main theorem. In this section we will offer a proof of our main result, Theorem (1.1).

We first give some definitions. Let $0<R_{0}<R_{1} \leq+\infty$ be fixed and suppose that $f_{1}, \cdots, f_{m}$ are $m \geq 2$ functions which are analytic and have no common zeros on $R_{0} \leq|z|<R_{1}$. Then $F=\left\{f_{1}, \cdots, f_{m}\right\}$ is called an analytic curve on $R_{0} \leq$ $|z|<R_{1}$ or simply an analytic curve. For $R_{0} \leq|z|<R_{1}$ we define $U(z, F)=$ $U\left(z ; f_{1}, \cdots, f_{m}\right)$ by the condition that

$$
U(z, F)=\max \left\{\left|f_{1}(z)\right|, \cdots,\left|f_{m}(z)\right|\right\} .
$$


Next for $R_{0} \leq R<R_{1}$ we define $T_{0}(R, F)=T_{0}\left(R ; f_{1}, \cdots, f_{m}\right)$ by the condition that

$$
T_{0}(R, F)=\frac{1}{2 \pi} \int_{0}^{2 \pi} \log U\left(R e^{i \theta}, F\right) d \theta
$$

The expression $T_{0}(R, F)$ is called the characteristic function of the analytic curve $F$. This expression is not necessarily different from the characteristic introduced for meromorphic curves by H. Cartan in [3].

For $a>0$ and $b \geq 0$ we note that $\max \{a, b\}=a(b / a)^{+}$so that $\log \max \{a, b\}=$ $\log a+\log ^{+}(b / a)$. From this identity and from Theorem (2.1), the Jensen integral formula for an annulus, the following identity is easily verified.

Lemma (3.1). Let $f$ and $g$ be analytic and have no common zeros on $0<$ $R_{0} \leq|z|<R_{1} \leq+\infty$ and suppose that $g(z) \neq 0$ for $|z|=R_{0}$. Then

$$
T(R, f / g)=T_{0}(R ; f, g)-\frac{1}{2 \pi} \int_{0}^{2 \pi} \log \left|g\left(R_{0} e^{i \theta}\right)\right| d \theta-\frac{1}{2 \pi} \Delta_{R_{0}} \arg g \log \frac{R}{R_{0}},
$$

for $R_{0} \leq R<R_{1}$

As an immediate corollary we obtain the following result.

Corollary (3.1). Let $F=\left\{f_{1}, \cdots, f_{m}\right\}$ be an analytic curve on $0<R_{0} \leq$ $|z|<R_{1} \leq+\infty$ and suppose that $f_{m}(z) \neq 0$ for $|z|=R_{0}$. Then

$$
T\left(R, f_{k} / f_{m}\right) \leq T_{0}(R, F)-\frac{1}{2 \pi} \int_{0}^{2 \pi} \log \left|f_{m}\left(R_{0} e^{i \theta}\right)\right| d \theta-\frac{1}{2 \pi} \Delta_{R_{0}} \arg f_{m} \log \frac{R}{R_{0}},
$$

for $R_{0} \leq R<R_{1}$ and $k=1, \cdots, m-1$.

We are particularly interested in the above result when $f_{m}$ is a polynomial. The following result is readily verified.

Corollary (3.2). Let $F=\left\{f_{1}, \cdots, f_{m}\right\}$ be an analytic curve on $1<R_{0} \leq$ $|z|<R_{1} \leq+\infty$ and suppose that $f_{m}$ is a polynomial such that $\left|f_{m}(z)\right| \geq 1$ for $|z|=R_{0}$. Then $T_{0}(R, F) \geq 0$ for $R_{0} \leq R<R_{1}$. Also there is some positive constant $A$ so that

$$
T\left(R, f_{k}\right)<A \log R+T_{0}(R, F), \text { for } R_{0} \leq R<R_{1} \text { and } k=1,2, \cdots, m \text {. }
$$

We now have the following result which will be of service in the discussion to follow.

Lemma (3.2). Let $F=\left\{f_{1}, \cdots, f_{m}\right\}$ be an analytic curve on $1<R_{0} \leq$ $|z|<+\infty$ and let $f_{m}$ be a polynomial such that $\left|f_{m}(z)\right| \geq 1$ for $|z|=R_{0}$. Suppose there are positive constants $B$ and $C$ and suppose there is an increasing sequence $\left\{R_{j}\right\}, j=1,2, \cdots$, of real numbers greater than $R_{0}$ and diverging to $+\infty$ such that 


$$
T_{0}\left(R_{j}, F\right)<B \log R_{j}+C \log ^{+} T_{0}\left(R_{j}, F\right),
$$

for $j=1,2, \cdots$. Then the $(m-1) \geq 1$ functions $f_{1}, \cdots, f_{m-1}$ do not have an essential isolated singularity at the point infinity.

Proof. Since $(\log x) / x \rightarrow 0$ as $x \rightarrow+\infty$ there is some $x_{0}>0$ so that $C \log ^{+} x<x / 2$ whenever $x>x_{0}$. Hence

$$
T_{0}\left(R_{j}, F\right)<\left(2 B+x_{0} / \log R_{0}\right) \log R_{j} \quad \text { for } j=1,2, \cdots \text {. }
$$

From the above estimate and from Corollary (3.2) we see that there is some positive constant $D$ so that $T\left(R_{j}, f_{k}\right)<D \log R_{j}$ for $j=1,2, \cdots$, and $k=1,2, \cdots$, $m-1$. From Corollary (2.1) it follows that the functions $f_{1}, \cdots, f_{m-1}$ do not have an essential isolated singularity at the point infinity. This proves the result.

We next need the following preliminary result.

Lemma (3.3). Let $f$ be polyanalytic on some nonempty subset $A$ of $\Gamma$ and suppose that $f$ is represented on $A$ by means of the equation $f(z)=$ $\sum_{k=0}^{n}(z \bar{z})^{k} f_{k}(z)$, where $f_{0}, \cdots, f_{n}$ are analytic on $A$, and where $f_{n} \not \equiv$ on $A$. Then $f$ may be represented on $A$ by means of the equation

$$
f(z)=\sum_{k=1}^{m} P_{k}(z \bar{z}) g_{k}(z),
$$

where for $k=1, \cdots, m$ the $P_{k}$ are monic polynomials of degree $d_{k}$ such that $0 \leq d_{1}<d_{2}<\cdots<d_{m}$, where $g_{1}, \cdots, g_{m}$ are anulytic and linearly independent on $A$, where each one of the functions $g_{1}, \cdots, g_{m}$ is one of the functions $f_{0}, \cdots, f_{n}$, and where $g_{m}=f_{n}$.

A proof of the above result can readily be established by induction on $n \geq 0$ [1].

Now let $0 \leq|z|<+\infty$ be fixed and let $f$ be polyanalytic on $R_{1} \leq|z|<+\infty$, that is on $A\left(\infty, 1 / R_{1}\right)$, and admit the exceptional value zero at the point infinity. Suppose further that $f$ is represented on $A\left(\infty, 1 / R_{1}\right)$ by means of equation (1.1) where $f_{n} \equiv 1$ on $A\left(\infty, 1 / R_{1}\right)$. From Lemma (3.3) it is easy to see that $f$ may be represented on $R_{1} \leq|z|<+\infty$ by means of the equation

$$
f(z)=b(z) g(z)
$$

where $h$ is analytic and never zero on $R_{1} \leq|z|<+\infty$, and where $g$ is polyanalytic on $R_{1} \leq|z|<+\infty$ and represented on $R_{1} \leq|z|<+\infty$ by means of the equation 


$$
g(z)=\sum_{k=1}^{m} P_{k}(z \bar{z}) g_{k}(z),
$$

where for $k=1, \cdots, m$ the $P_{k}$ are monic polynomials of degree $d_{k}$ such that $0 \leq d_{1}<d_{2}<\cdots<d_{m}$, where $g_{1}, \cdots, g_{m}$ are analytic and linearly independent on $R_{1} \leq|z|<+\infty$, and where $g_{m}$ is a nonidentically zero polynomial.

Note that $g$ admits the exceptional value zero at the point infinity. Now in order to establish Theorem (1.1) it is only necessary to show that $g$ does not have an essential isolated singularity at the point infinity, that is it is only necessary to show that the functions $g_{1}, \cdots, g_{m-1}$ do not have an essential isolated singularity at the point infinity.

Now if $m=1$ in (3.4), then Theorem (1.1) follows trivally. Hence in the discussion to follow we shall assume that $m \geq 2$.

There is some $R_{0}$ with $R_{0} \geq R_{1}$ so that $g$ never vanishes on $R_{0} \leq|z|<+\infty$. It therefore follows that the $m \geq 2$ functions $g_{1}, \cdots, g_{m}$ are analytic, linearly independent, and have no common zeros on $R_{0} \leq|z|<+\infty$.

At this point it will be convenient to impose several further conditions on $R_{0}$. First note that $g_{m}$ is a nonidentically zero polynomial. From (3.3), (3.4) we see that we may assume that $g_{m}$ is a polynomial of degree at least $m$. We may now assume that $R_{0}$ with $R_{0} \geq R_{1}$ is so chosen that $\left|g_{m}(z)\right| \geq 1$ for $|z|=R_{0}$ and $\left|g_{m}^{(k)}(z)\right| \neq 0$ for $|z|=R_{0}$ and $k=1, \ldots, m-1$. Second note that $g_{1}, \ldots, g_{m}$ are analytic and linearly independent on $R_{1} \leq|z|<+\infty$. Hence the Wronskian $W\left(g_{1}, \cdots, g_{m}\right)$ of $g_{1}, \cdots, g_{m}$ does not vanish identically on $R_{1} \leq|z|<+\infty$. We may now assume that $R_{0}$ with $R_{0} \geq R_{1}$ is so chosen that $W\left(g_{1}, \cdots, \mathrm{g}_{m}\right)$ does not vanish on $|z|=R_{0}$. Finally we shall assume that $R_{0}>1$. Such a value of $R_{0}$ once determined will remain fixed for the remainder of this section.

Now for each $\rho \geq R_{0}$ we define $g(z, \rho)$ on $R_{0} \leq|z|<+\infty$ by the condition that

$$
g(z, \rho)=\sum_{k=1}^{m} P_{k}\left(\rho^{2}\right) g_{k}(z) .
$$

Observe for each $\rho \geq R_{0}$ that $g(z, \rho)$ is analytic on $R_{0} \leq|z|<+\infty$ and that $g(z, \rho)=g(z)$ for $|z|=\rho$. Note also that for each $\rho \geq R_{0}$ that $g(z, \rho)$ is a certain linear combination of the $m \geq 2$ funcţions $g_{1}, \cdots, g_{m}$ which are analytic, linearly independent, and with no common zeros on $R_{0} \leq|z|<+\infty$.

Let $a_{1}, a_{2}, \ldots, a_{m+1}$ be $(m+1) \geq 3$ distinct real numbers such that

$$
2<a_{1}<a_{2}<\cdots<a_{m+1}<3 .
$$

These $(m+1)$ real numbers $a_{1}, a_{2}, \ldots, a_{m+1}$ once chosen will remain fixed 
throughout the remainder of this section.

In order to keep our notation reasonably compact we let

$$
\rho_{\mu}=a_{\mu} \rho \quad(\mu=1,2, \cdots, m+1),
$$

for $\rho \geq R_{0}$. We also let

$$
G_{\mu}=g\left(z, \rho_{\mu}\right) \quad(\mu=1,2, \cdots, m+1),
$$

for $\rho \geq R_{0}$ and $R_{0}|z|<+\infty$.

From (3.5), (3.8) we obtain

$$
G_{\mu}=\sum_{k=1}^{m} P_{k}\left(\rho_{\mu}^{2}\right) g_{k}(z) \quad(\mu=1,2, \cdots, m+1),
$$

for $\rho \geq R_{0}$ and $R_{0} \leq|z|<+\infty$.

Next let $\beta_{1}, \beta_{2}, \cdots, \beta_{m}$ be a sequence of $m$ distinct integers selected in any manner from the $(m+1)$ integers $1,2, \cdots, m+1$. Such a sequence of $m$ integers $\beta_{1}, \beta_{2}, \cdots, \beta_{m}$ formed in this manner will be termed admissible.

From (3.9) we see that

$$
G_{\beta_{\mu}}=\sum_{k=1}^{m} P_{k}\left(\rho_{\beta_{\mu}}^{2}\right) g_{k}(z) \quad(\mu=1,2, \cdots, m),
$$

for $\rho \geq R_{0}$ and $R_{0} \leq|z|<+\infty$ and for all admissible $\beta_{1}, \beta_{2}, \cdots, \beta_{m}$.

We now assume that $\sigma_{0}$ with $\sigma_{0} \geq R_{0}$ is so chosen that $P_{k}\left(\rho^{2}\right) \neq 0$ for $\rho \geq \sigma_{0}$ and $k=1,2, \cdots, m$.

Hence (3.10) can be written as

$$
G_{\beta_{\mu}}=\sum_{k=1}^{m}\left[\frac{P_{k}\left(\rho_{\beta_{\mu}}^{2}\right)}{P_{k}\left(\rho^{2}\right)}\right] P_{k}\left(\rho^{2}\right) g_{k}(z) \quad(\mu=1,2, \cdots, m),
$$

for $\rho \geq \sigma_{0}$ and $R_{0} \leq|z|<+\infty$ and for all admissible $\beta_{1}, \beta_{2}, \cdots, \beta_{m}$.

For all $\rho \geq \sigma_{0}$ and for all admissible $\beta_{1}, \beta_{2}, \cdots, \beta_{m}$ let

$$
C\left(\beta_{1}, \cdots, \beta_{m} ; \rho\right)
$$

denote the determinant of order $m$ of the matrix with $P_{k}\left(\rho_{\beta_{\mu}}^{2}\right)$ as the entry in the $k$ th column and the $\mu$ th row for $k, \mu=1,2, \cdots, m$. Again for $\rho \geq \sigma_{0}$ and admissible $\beta_{1}, \beta_{2}, \cdots, \beta_{m}$ let

$$
D\left(\beta_{1}, \ldots, \beta_{m} ; \rho\right)
$$

denote the determinant of order $m$ of the matrix with $P_{k}\left(\rho_{B_{\mu}}^{2}\right) / P_{k}\left(\rho^{2}\right)$ as the entry in the $k$ th column and the $\mu$ th row for $k, \mu=1,2, \cdots, m$. Finally for all admissible $\beta_{1}, \beta_{2}, \cdots, \beta_{m}$ let 


$$
\sigma\left(\beta_{1}, \cdots, \beta_{m}\right)=\left|\begin{array}{cccc}
a_{\beta_{1}}^{2 d_{1}}, & a_{\beta_{1}}^{2 d_{2}}, & \cdots, & a_{\beta_{1}}^{2 d_{m}} \\
a_{\beta_{2}}^{2 d_{1}}, & a_{\beta_{2}}^{2 d_{2}}, & \cdots, & a_{\beta_{2}}^{2 d_{m}} \\
\cdot & & & \\
\cdot & & & \\
a_{\beta_{m}}^{2 d_{1}}, & a_{\beta_{m}}^{2 d_{2}}, & \cdots, & a_{\beta_{m}}^{2 d_{m}}
\end{array}\right|
$$

be the determinant of order $m$ of the matrix with $a_{B_{\mu}}^{2 d_{k}}$ as the entry in the $k$ th column and the $\mu$ th row for $k, \mu=1,2, \cdots, m_{\text {. Here }} a_{1}, a_{2}, \cdots, a_{m+1}$ satisfy (3.6) and $d_{k}$ is the degree of the polynomial $P_{k}$ for $k=1,2, \cdots, m$.

It is not difficult to be persuaded that $\sigma\left(\beta_{1}, \cdots, \beta_{m}\right) \neq 0$ for all admissible $\beta_{1}, \beta_{2}, \cdots, \beta_{m}$. To see this fix $a_{\beta_{2}}, \cdots, a_{\beta_{m}}$. Then $\sigma\left(\beta_{1}, \cdots, \beta_{m}\right)$ can be regarded as a polynomial in $a_{\beta_{1}}$. Evidently $a_{\beta_{2}}, \ldots, a_{\beta_{m}}$ are $(m-1)$ distinct positive real roots of $\sigma\left(\beta_{1}, \cdots, \beta_{m}\right)$. If we now expand the determinant in (3.14) by means of the first row we deduce from Descarte's rule of signs that $\sigma\left(\beta_{1}, \cdots, \beta_{m}\right)$ can have at most $(m-1)$ positive real roots. Consequently $\sigma\left(\beta_{1}, \cdots, \beta_{m}^{m}\right) \neq 0$ as asserted.

Now for $k=1,2, \cdots, m$ the polynomials $P_{k}$ are monic and of degree $d_{k} \geq 0$. From (3.7) we deduce that $P_{k}\left(\rho_{\mu}^{2}\right) / P_{k}\left(\rho^{2}\right)^{k} \rightarrow a_{\mu}^{2 d_{k}}$ as $\rho \rightarrow+\infty$ for $k=$ $1,2, \cdots, m$ and $\mu=1,2, \cdots, m+1$. From the above observation and from (3.13), (3.14) we see that $D\left(\beta_{1}, \cdots, \beta_{m} ; \rho\right) \rightarrow \sigma\left(\beta_{1}, \cdots, \beta_{m}\right) \neq 0$ as $\rho \rightarrow+\infty$ for all admissible $\beta_{1}, \beta_{2}, \cdots, \beta_{m}$. Hence we may assume that $\sigma_{0}$ with $\sigma_{0} \geq R_{0}$ is chosen so large that

$$
\left|D\left(\beta_{1}, \cdots, \beta_{m} ; \rho\right)\right|>D
$$

for all $\rho \geq \sigma_{0}$ and for all admissible $\beta_{1}, \beta_{2}, \cdots, \beta_{m}$, where $D$ is some positive constant. From (3.12), (3.13) we see that

$$
C\left(\beta_{1}, \cdots, \beta_{m} ; \rho\right)=D\left(\beta_{1}, \cdots, \beta_{m} ; \rho\right) P_{1}\left(\rho^{2}\right) P_{2}\left(\rho^{2}\right) \ldots P_{m}\left(\rho^{2}\right)
$$

for all $\rho \geq \sigma_{0}$ and for all admissible $\beta_{1}, \beta_{2}, \cdots, \beta_{m}$. From this identity and from (3.15) we see that $\sigma_{0}$ with $\sigma_{0} \geq R_{0}$ can be chosen so large that

$$
\left|C\left(\beta_{1}, \cdots, \beta_{m} ; \rho\right)\right|>1,
$$

for all $\rho \geq \sigma_{0}$ and for all admissible $\beta_{1}, \beta_{2}, \cdots, \beta_{m}$.

In view of (3.10), (3.16) we see that for $\rho \geq \sigma_{0}$ that any $m$ of the $(m+1)$ functions $G_{1}, G_{2}, \cdots, G_{m+1}$ are analytic linearly independent, and have no common zeros on $R_{0} \leq|z|<+\infty$. 
Now let $G$ denote the analytic curve.

$$
G=\left\{g_{1}, \cdots, g_{m}\right\},
$$

and for $\rho \geq \sigma_{0}$ let $G(\rho)$ denote the analytic curve

$$
G(\rho)=\left\{G_{1}, \cdots, G_{m+1}\right\} .
$$

From (3.1), (3.11), (3.15), (3.18) we see that there is some positive constant $A$ so that $\left|P_{k}\left(\rho^{2}\right) g_{k}(z)\right|<A U(z, G(\rho))$ for $\rho \geq \sigma_{0}$ and $R_{0} \leq|z|<+\infty$ and $k=$ $1,2, \cdots, m$. We may now assume that $\sigma_{0}$ with $\sigma_{0} \geq R_{0}$ is chosen so large that $\left|P_{k}\left(\rho^{2}\right)\right|>A$ for $\rho \geq \sigma_{0}$ and $k=1,2, \cdots, m$. Hence $\left|g_{k}(z)\right|<U(z, G(\rho))$ for $\rho \geq \sigma_{0}$ and $R_{0} \leq|z|<+\infty$ and $k=1,2, \cdots, m$. From the above estimate and from (3.1), (3.17) we see that $U(z, G)<U(z, G(\rho))$ for $\rho \geq \sigma_{0}$ and $R_{0} \leq|z|<+\infty$. From the above estimate and from (3.2) we see that

$$
T_{0}(R, G)<T_{0}(R, G(\rho)),
$$

for $\rho \geq \sigma_{0}$ and $R \geq R_{0}$.

Next from (3.7), (3.9) we see that

$$
\lim _{\rho \rightarrow+\infty} \frac{G_{\mu}^{(k)}}{P_{m}\left(\rho_{\mu}^{2}\right)}=g_{m}^{(k)},
$$

uniformly with respect to $z$ on $|z|=R_{0}$ for $k=0,1, \cdots, m-1$ and $\mu=1$, $2, \cdots, m+1$. From the above equation we see that $\sigma_{0}$ with $\sigma_{0} \geq R_{0}$ may be chosen so large that $G_{\mu}^{(k)} \neq 0, \infty$ for $|z|=R_{0}$ and $k=0,1, \cdots, m-1$ and $\mu=$ $1,2, \cdots, m+1$, whenever $\rho \geq \sigma_{0}$. Also we see that $\sigma_{0}$ with $\sigma_{0} \geq R_{0}$ may be chosen so large that there is some positive constant $A$ so that

$$
\frac{\left|G_{\mu}^{(k)}\right|+1}{\left|G_{\mu}^{(k-1)}\right|}<A,
$$

for $|z|=R_{0}$ and $k=1,2, \cdots, m-1$ and $\mu=1,2, \cdots, m+1$, whenever $\rho \geq \sigma_{0^{\circ}}$ Next for $\rho \geq \sigma_{0}$ define $H(z, \rho)$ on $R_{0} \leq .|z|<+\infty$ by the condition that

$$
H(z, \rho)=\frac{G_{1} G_{2} \cdots G_{m+1}}{W\left(g_{1}, \cdots, g_{m}\right)} .
$$

Evidently $H(z, \rho)$ is meromorphic on $R_{0} \leq|z|<+\infty$ and $H(z, \rho) \neq 0, \infty$ for $|z|=R_{0}$ whenever $\rho \geq \sigma_{0}$.

Since $g$ never vanishes on $R_{0} \leq|z|<+\infty$ there is some integer $p$ so that $\Delta_{\rho}$ arg $g=2 \pi p$ for all $\rho \geq \mathrm{R}_{0}$. Hence from (3.5) we see that $\Delta_{\rho}$ arg $g(z, \rho)=2 \pi p$ for all $\rho \geq R_{0}$. Since $g_{m}(z) \neq 0$ for $|z|=R_{0}$, there is some integer $q$ so that $\Delta_{R_{0}}$ arg $g_{m}=2 \pi q$. From (3.5) we see that $g(z, \rho) / P_{m}\left(\rho^{2}\right) \rightarrow g_{m}(z)$ as $\rho \rightarrow+\infty$ uniformly with respect to $z$ on $|z|=R_{0}$. Hence we may assume that $\sigma_{0}$ with 
$\sigma_{0} \geq R_{0}$ is chosen so large that $\Delta_{R_{0}}$ arg $g(z, \rho)=2 \pi q$ for $\rho \geq \sigma_{0}$. Consequently $\Delta_{\rho}$ arg $g(z, \rho)-\Delta_{R_{0}}$ arg $g(z, \rho)=2 \pi(p-q)$ for $\rho \geq \sigma_{0}$. Thus for $\rho \geq \sigma_{0}$ we see that $g(z, \rho)$ has exactly $s=(p-q)$ zeros, counting multiplicities, on $R_{0}<|z| \leq \rho$. Thus for $\mu=1,2, \cdots, m+1$ we see that if $\rho_{\mu} \geq \sigma_{0}$ then $G_{\mu}=g\left(z, \rho_{\mu}\right)$ has $s$ zeros on $R_{0}<|z| \leq \rho_{\mu}$. From (3.6), (3.7) we see that $2 \rho<\rho_{\mu}$ for $\rho>0$ and $\mu=$ $1,2, \cdots, m+1$. Thus for $\rho \geq \sigma_{0}$ and $\mu=1,2, \cdots, m+1$ we see that $G_{\mu}$ has at most $s$ zeros on $R_{0}<|z| \leq R$ for all $R \geq R_{0}$ provided that $R \leq 2 \rho$. Hence there is some positive constant $A$ so that $N\left(R, R_{0}, 1 / G_{\mu}\right)<A \log R$, for $\rho \geq \sigma_{0}$ and $R_{0} \leq R \leq 2 \rho$ and for $\mu=1,2, \cdots, m+1$. From (3.21) we see that

$$
N\left(R, R_{0}, \frac{1}{H(z, \rho)}\right) \leq \sum_{\mu=1}^{m+1} N\left(R, R_{0}, 1 / G_{\mu}\right),
$$

for $\rho \geq \sigma_{0}$ and $R \geq R_{0}$. From the above two inequalities we see that there is some positive constant $A$ so that

$$
N\left(R, R_{0}, 1 / H(z, \rho)\right)<A \log R,
$$

for $\rho \geq \sigma_{0}$ and $R_{0} \leq R \leq 2 \rho$. Next from (3.7), (3.9), (3.21) we see that

$$
\lim _{\rho \rightarrow+\infty} \frac{H(z, \rho)}{P_{m}\left(\rho_{1}\right) \cdots P_{m}\left(\rho_{m+1}\right)}=\frac{g_{m}^{m+1}}{W\left(g_{1}, \cdots, g_{m}\right)},
$$

uniformly with respect to $z$ on $|z|=R_{0}$. Hence we may assume that $\sigma_{0}$ with $\sigma_{0} \geq R_{0}$ is chosen so large that there is some positive constant $A$ and some integer $p$ so that

$$
\log |H(z, \rho)|<A \log \rho, \quad \Delta_{R_{0}} \arg H(z, \rho)=2 \pi p,
$$

for $|z|=R_{0}$ and $\rho \geq \sigma_{0}$.

In the foregoing discussion we have chosen $\sigma_{0}$ with $\sigma_{0} \geq R_{0}>1$ so as to satisfy a number of conditions. This $\sigma_{0}$ will now remain fixed for the remainder of this section.

Now from Theorem (1.2), the Jensen integral formula for an annulus, we see that

$$
\begin{aligned}
\frac{1}{2 \pi} \int_{0}^{2 \pi} \log \left|H\left(R e^{i \theta}, \rho\right)\right| d \theta= & N\left(R, R_{0}, \frac{1}{H(z, \rho)}\right)-N\left(R, R_{0}, H(z, \rho)\right) \\
& +\frac{1}{2 \pi} \int_{0}^{2 \pi} \log \left|H\left(R_{0} e^{i \theta}\right)\right| d \theta+\frac{1}{2 \pi} \Delta_{R_{0}} \arg H(z, \rho) \log \frac{R}{R_{0}},
\end{aligned}
$$

for $\rho \geq \sigma_{0}$ and $R \geq R_{0}$. From the above identity and from (3.22), (3.23) we see that there are positive constants $A$ and $B$ so that 


$$
\frac{1}{2 \pi} \int_{0}^{2 \pi} \log \left|H\left(R e^{i \theta}, \rho\right)\right| d \theta<A \log R+B \log \rho
$$

for $\rho \geq \sigma_{0}$ and $R_{0} \leq R \leq 2 \rho$.

Next for all admissible $\beta_{1}, \beta_{2}, \cdots, \beta_{m}$ let $W\left(G_{\beta_{1}}, \cdots, G_{\beta_{m}}\right)$ denote the Wronskian of $G_{\beta_{1}}, G_{\beta_{2}}, \cdots, G_{\beta_{m}}$ For $\rho \geq \sigma_{0}$ and $R_{0} \leq|z|<+\infty$ let $V(z, \rho)$ denote the maximum of the absolute value of the expression

$$
\frac{W\left(G_{\beta_{1}}, \cdots, G_{\beta_{m}}\right)}{G_{\beta_{1}} \cdots G_{\beta_{m}}}=\left|\begin{array}{cccc}
1, & 1, & \cdots, & 1 \\
\frac{G_{\beta_{1}}^{\prime}}{G_{\beta_{1}}}, & \frac{G_{\beta_{2}}^{\prime}}{G_{\beta_{2}}}, & \cdots, & \frac{G_{\beta_{m}}}{G_{\beta_{m}}} \\
\frac{G_{\beta_{1}}^{\prime \prime}}{G_{\beta_{1}}}, & \frac{G_{\beta_{2}}^{\prime \prime}}{G_{\beta_{2}}}, \cdots, & & \frac{G_{\beta_{m}}^{\prime \prime}}{G_{\beta_{m}}} \\
\vdots & & & \\
\frac{G_{\beta_{1}}^{(m-1)}}{G_{\beta_{1}}}, & \frac{G_{\beta_{2}}^{(m-1)}}{G_{\beta_{2}}}, \cdots, & \frac{G_{\beta_{m}}^{(m-1)}}{G_{\beta_{m}}}
\end{array}\right|
$$

for all admissible $\beta_{1}, \beta_{2}, \cdots, \beta_{m}$.

It is easy to see that there are positive constants $A$ and $B$ so that

$$
\frac{1}{2 \pi} \int_{0}^{2 \pi} \log V\left(R e^{i \theta}, \rho\right) d \theta<A+B \sum_{\mu=1}^{m+1} \sum_{k=1}^{m-1} m\left(R, G_{\mu}^{(k)} / G_{\mu}\right),
$$

for $\rho \geq \sigma_{0}$ and $R \geq R_{0}$. However $m\left(R, G_{\mu}^{(k)} / G_{\mu}\right) \leq \Sigma_{j=1}^{k} m\left(R, G_{\mu}^{(j)} / G_{\mu}^{(j-1)}\right)$, for $\rho \geq \sigma_{0}$ and $R \geq R_{0}$ and $k=1,2, \cdots, m-1$ and $\mu=1,2, \cdots, m+1$. From the two previous inequalities we see that there are positive constants $A$ and $B$ such that

$$
\frac{1}{2 \pi} \int_{0}^{2 \pi} \log V\left(R e^{i \theta}, \rho\right) d \theta<A+B \sum_{\mu=1}^{m+1} \sum_{k=1}^{m-1} m\left(R, G_{\mu}^{(k)} / G_{\mu}^{(k-1)}\right)
$$

for $\rho \geq \sigma_{0}$ and $R \geq R_{0}$.

From Theorem (2.4) we see that for $k=1,2, \cdots, m-1$ and $\mu=1,2, \cdots$, $m+1$ and for $a>0$ and $\rho \geq \sigma_{0}$ that

$$
\begin{aligned}
m\left(R, G_{\mu}^{(k)} / G_{\mu}^{(k-1)}\right) \leq & 4 \log ^{+} T\left(R, G_{\mu}^{(k-1)}\right)+3 \log R \\
& +4 \frac{1}{2 \pi} \int_{0}^{2 \pi} \frac{\left|G_{\mu}^{(k)}\left(R_{0} e^{i \theta}\right)\right|+1}{\left|G_{\mu}^{(k-1)}\left(R_{0} e^{i \theta}\right)\right|} d \theta+3 \log ^{+} \frac{1}{a}+17 \log 2,
\end{aligned}
$$


for all $R \geq R_{0}$ except perhaps for a set $I=I(a, \rho, k, \mu)$ of measure at most $a$. It is important to note that $I$ depends not only on $a$ but also on the function $G_{\mu}^{(k-1)}=g^{(k-1)}\left(z, a_{\mu} \rho\right)$. Thus we see that $I$ depends on the parameters $a, \rho, k, \mu$. From the above estimate and from (3.20) we see that there is some positive constant $A$ so that for $k=1,2, \cdots, m-1$ and $\mu=1,2, \cdots, m+1$ and for $a>0$ and $\rho \geq \sigma_{0}$ that

$$
m\left(R, G_{\mu}^{(k)} / G_{\mu}^{(k-1)}\right)<4 \log ^{+} T\left(R, G_{\mu}^{(k-1)}\right)+A \log R+3 \log ^{+} \frac{1}{a},
$$

for all $R \geq R_{0}$ except perhaps for a set $I=I(a, \rho, k, \mu)$ of measures at most $a$. However

$$
T\left(R, G_{\mu}^{(k-1)}\right) \leq \sum_{j=1}^{k-1} m\left(R, \frac{G_{\mu}^{(j)}}{G_{\mu}^{(j-1)}}\right)+T\left(R, G_{\mu}\right)
$$

for $\rho \geq \sigma_{0}$ and $R \geq R_{0}$ and for $k=1,2, \cdots, m-1$ and $\mu=1,2, \cdots, m+1$. From the above two estimates we see that there is some positive constant $A$ so that for $k=1,2, \cdots, m-1$ and $\mu=1,2, \cdots, m+1$ and for $a>0$ and $\rho \geq \sigma_{0}$

$$
m\left(R, \frac{G_{\mu}^{(k)}}{G_{\mu}^{(k-1)}}\right)<4 \log ^{+} T\left(R, G_{\mu}\right)+A \log R+3 \log ^{+} \frac{1}{a}+4 \sum_{j=1}^{k-1} m\left(R, \frac{G_{\mu}^{(j)}}{G_{\mu}^{(j-1)}}\right)
$$

for all $R \geq R_{0}$ except perhaps for a set $I=I(a, \rho, k, \mu)$ of measure at most $a$. From the above recursive inequality we deduce that for $k=1,2, \cdots, m-1$ and $\mu=1,2, \cdots, m+1$ and for $a>0$ and $\rho \geq \sigma_{0}$ that

$$
m\left(R, \frac{G_{\mu}^{(k)}}{G_{\mu}^{(k-1)}}\right)<5^{k-1}\left[4 \log ^{+} T\left(R, G_{\mu}\right)+A \log R+3 \log ^{+} 1 / a\right]
$$

for all $R \geq R_{0}$ except perhaps for a set $I=I(a, \rho, k, \mu)$ of measure at most $k a$. Consequently for $\mu=1,2, \cdots, m+1$ and for $a>0$ and $\rho \geq \sigma_{0}$ we see that

$$
\sum_{k=1}^{m-1} m\left(R, \frac{G_{\mu}^{(k)}}{G_{\mu}^{(k-1)}}\right)<\frac{5^{m-1}}{4}\left[4 \log ^{+} T\left(R, G_{\mu}\right)+A \log R+3 \log ^{+} 1 / a\right]
$$

for all $R \geq R_{0}$ except perhaps for a set $I=I(a, \rho, \mu)$ of measure at most $m(m-1) a / 2$. Hence for $a>0$ and $\rho \geq \sigma_{0}$ we see that 


$$
\begin{aligned}
\sum_{\mu=1}^{m+1} \sum_{k=1}^{m-1} m\left(R, \frac{G_{\mu}^{(k)}}{G_{\mu}^{(k-1)}}\right)< & 5^{m-1} \sum_{\mu=1}^{m+1} \log ^{+} T\left(R, G_{\mu}\right) \\
& +\frac{(m+1) 5^{m-1}}{4}\left[A \log R+3 \log ^{+} 1 / a\right]
\end{aligned}
$$

for all $R \geq R_{0}$ except perhaps for a set $I=I(a, \rho)$ of measure at most $(m+1) m(m-1) a / 2$. Hence there exist positive constants $A, B$ and $C$ so that for $a>0$ and $\rho \geq \sigma_{0}$ we have that

$$
\sum_{\mu=1}^{m+1} \sum_{k=1}^{m-1} m\left(R, \frac{G_{\mu}^{(k)}}{G_{\mu}^{(k-1)}}\right)<A \log R+B \log ^{+} \frac{1}{a}+C \sum_{\mu=1}^{m+1} \log ^{+} T\left(R, G_{\mu}\right)
$$

for all $R \geq R_{0}$ except perhaps for a set $I=I(a, \rho)$ of measure at most $a$. From the above inequality and from (3.26) we see that there are positive constants $A$, $B$, and $C$ so that for $a>0$ and $\rho \geq \sigma_{0}$ we have that

$$
\frac{1}{2 \pi} \int_{0}^{2 \pi} \log V\left(R e^{i \theta}, \rho\right) d \theta<A \log R+B \log ^{+} \frac{1}{a}+C \sum_{\mu=1}^{m+1} \log ^{+} T\left(R, G_{\mu}\right)
$$

for all $R \geq R_{0}$ except perhaps for a set $I=I(a, \rho)$ of measure at most $a$. Now from (2.17), (3.7), (3.9) we see that there is some positive constant $A$ so that $T\left(R, G_{\mu}\right)<A \log \rho+\Sigma_{k=1}^{m} T\left(R, g_{k}\right)$ for $\rho \geq \sigma_{0}$ and $R \geq R_{0}$ and for $\mu=1,2, \cdots$, $m+1$. On the other hand we see from Corollary (3.2) that there is some positive constant $A$ so that $T\left(R, g_{k}\right)<A \log R+T_{0}(R, G)$ for $R \geq R_{0}$ and $k=1,2, \cdots, m_{\text {. }}$

From the three previous inequalities we see that there are positive constants $A, B, C$, and $D$ so that for $a>0$ and $\rho \geq \sigma_{0}$ we have that

$$
\begin{aligned}
\frac{1}{2} \int_{0}^{2 \pi} & \log V\left(R e^{i \theta}, \rho\right) d \theta \\
& <A \log R+B \log \rho+C \log ^{+} 1 / a+D \log ^{+} T_{0}(R, G),
\end{aligned}
$$

for all $R \geq R_{0}$ except perhaps for a set $l=I(a, \rho)$ of measure at most $a$

Now from (3.10), (3.12) we see that

$$
W\left(G_{\beta_{1}}, \cdots, G_{\beta_{m}}\right)=C\left(\beta_{1}, \cdots, \beta_{m} ; \rho\right) W\left(g_{1}, \cdots, g_{m}\right),
$$

for $\rho \geq \sigma_{0}$ and $R_{0} \leq|z|<+\infty$ and for all admissible $\beta_{1}, \beta_{2}, \cdots, \beta_{m}$. Now from the above identity and from (3.21) we deduce that

$$
\frac{G_{\beta}}{H(z, \rho)}=\frac{1}{C\left(\beta_{1}, \cdots, \beta_{m} ; \rho\right)} \frac{W\left(G_{\beta_{1}}, \cdots, G_{\beta_{m}}\right)}{G_{\beta_{1}} \cdots G_{\beta_{m}}},
$$


for $\rho \geq \sigma_{0}$ and $R_{0} \leq|z|<+\infty$ and for all admissible $\beta_{1}, \beta_{2}, \cdots, \beta_{m}$, where $\beta$ is that unique integer from the $(m+1)$ integers $1,2, \cdots, m+1$ which is not among the integers $\beta_{1}, \beta_{2}, \cdots, \beta_{m}$. From the above identity and from (3.16), (3.25) we see that $\left|G_{\beta}\right| \leq|H(z, \rho)| V(z, \rho)$ for $\rho \geq \sigma_{0}$ and $R_{0} \leq|z|<+\infty$ and for $\beta=1$, $2, \cdots, m+1$. From the above estimate and from (3.1), (3.18) we see that $U(z, G(\rho)) \leq|H(z, \rho)| V(z, \rho)$ for $\rho \geq \sigma_{0}$ and $R_{0} \leq|z|<+\infty$. From the above estimate and from (3.2) we deduce that

$$
T_{0}(R, G(\rho)) \leq \frac{1}{2 \pi} \int_{0}^{2 \pi} \log \left|H\left(R e^{i \theta}, \rho\right)\right| d \theta+\frac{1}{2 \pi} \int_{0}^{2 \pi} \log V\left(R e^{i \theta}, \rho\right) d \theta
$$

for $\rho \geq \sigma_{0}$ and $R \geq R_{0}$.

From (3.19), (3.24), (3.27), (3.28) we see that there are positive constants $A, B, C$, and $D$ so that for $a>0$ and $\rho \geq \sigma_{0}$ we have that

$$
T_{0}(R, G)<A \log R+B \log \rho+C \log ^{+} 1 / a+D \log ^{+} T_{0}(R, G),
$$

for all $R_{0} \leq R \leq 2 \rho$ except perhaps for a set $I=I(a, \rho)$ of measure at most $a$. Hence there are positive constants $A, B$, and $C$ so that for $a>0$ and $\rho \geq \sigma_{0}$ we have that

$$
T_{0}(R, G)<A \log R+B \log ^{+} 1 / a+C \log ^{+} T_{0}(R, G)
$$

for all $\rho \leq R \leq 2 \rho$ except perhaps for a set $I=I(a, \rho)$ of measure at most $a$. Now let $a>0$ be fixed. Let $\sigma_{k}=2^{k} \sigma_{0}$ and $a_{k}=a / 2^{k+1}$ for $k=0,1, \ldots$. Hence we see that

$$
T_{0}(R, G)<A \log R+B \log ^{+} 1 / a_{k}+C \log ^{+} T_{0}(R, G)
$$

for $\sigma_{k} \leq R \leq \sigma_{k+1}$ except perhaps for a set $I_{k}$ of measure at most $a_{k}$ for $k=$ $0,1, \cdots$. Now for $k=0,1, \cdots$ we see that if $\sigma_{k} \leq R \leq \sigma_{k+1}$ then $1 / a_{k}=$ $2^{k+1} / a=2 \sigma_{k} / \sigma_{0} a \leq 2 R / \sigma_{0} a$. Thus for $k=0,1, \cdots$ we see that if $\sigma_{k} \leq R \leq \sigma_{k+1}$ then $\log ^{+}\left(1 / a_{k}\right) \leq \log R+\log 2+\log ^{+}(1 / a)$. Hence we see that there are positive constants $A, B$, and $C$ so that

$$
T_{0}(R, G)<A \log R+B \log ^{+} 1 / a+C \log ^{+} T_{0}(R, G)
$$

for all $2^{k} \sigma_{0} \leq R \leq 2^{k+1} \sigma_{0}$ except perhaps for a set $I_{k}$ of measure at most $a / 2^{k+1}$ for $k=0,1, \cdots$. Hence we see that for all $a>0$

$$
T_{0}(R, G)<A \log R+B \log ^{+} 1 / a+C \log ^{+} T_{0}(R, G)
$$

for all $R \geq \sigma_{0}$ except perhaps for a set $I=I(a)$ of measure at most $a$. Hence there are positive constants $A$ and $B$ so that

$$
T_{0}(R, G)<A \log R+B \log ^{+} T_{0}(R, G)
$$


for all $R \geq R_{0}$ except perhaps for a set of finite measure.

From Lemma (3.2) and from (3.17), (3.29) we see that the functions $g_{1}, \ldots$, $g_{m-1}$ do not have an essential isolated singularity at the point infinity. From (3.3) and (3.4) we see that the functions $f_{0}, f_{1}, \cdots, f_{n-1}$ do not have an essential isolated singularity at the point infinity. This completes the proof of Theorem (1.2).

4. Applications. In this section we will give some simple applications of Theorem (1.1) and of the equivalent formulations of Theorem (1.1) given in Corollaries (1.1), (1.2), and (1.3).

First if in Corollary (1.2) we let $A\left(z_{0}, R\right)=\Gamma$, that is if we let $z_{0}=\infty$ and $R=+\infty$, we obtain the following result.

Corollary (4.1). Let $f$ be polyentire and given by equation (1.1) and suppose that $f$ admits the exceptional value zero at the point infinity. If $j=0,1, \cdots, n$ is such that $f_{j} \equiv 0$ then the function $f_{j}$ admits the exceptional value zero at the point infinity and for $i=0,1, \cdots, n$ the functions $f_{i} / f_{j}$ are rational functions.

Next if in Corollary (1.3) we let $A\left(z_{0}, R\right) \neq \Gamma$ we obtain the following representation due to M. B. Balk [1] for polyentire functions which admit an exceptional value at the point infinity.

Corollary (4.2). If $f$ is a polyentire function which admits the exceptional value zero at the point infinity, then there exists an entire function $b$ and $a$ polynomial $P(z, \bar{z})$ in $z$ and $\bar{z}$ such that $f(z)=e^{b(z)} P(z, \bar{z})$, for all $z \in \Gamma$.

First a comment concerning the representation of the polyanalytic function $f$ in Corollary (1.3) seems to be in order. If $A\left(z_{0}, R\right)=\Gamma$ then from Corollary (4.1) we see that the polyanalytic function $g$ in Corollary (1.3) may be assumed to be a polynomial in $z$ and $\bar{z}$. However if $A\left(z_{0}, R\right) \neq \Gamma$ then $g$ need not in general be a polynomial in $z$ and $\bar{z}$. Suppose that $f$ is represented on $A\left(z_{0}, R\right)$ by (1.1) where $f_{j} \not \equiv 0$ on $A\left(z_{0}, R\right)$ for some $j=0,1, \cdots, n$. Then it is easy to see that $g$ may be assumed to be a polynomial in $z$ and $\bar{z}$ if and only if for $i=0,1, \cdots, n$ the functions $f_{i} / f_{j}$ are rational functions.

As a further application of Theorem (1.1) we deduce the big Picard theorem for polyanalytic functions.

Corollary (4.3). Let $f$ be polyanalytic with an isolated singularity at the point $z_{0}$. If $f$ admits the exceptional values zero and one at the point $z_{0}$ then $f$ has a nonessential singularity at the point $z_{0}$.

Proof. Let $f$ be polyanalytic on $A\left(z_{0}, R\right)$ and represented on $A\left(z_{0}, R\right)$ by (1.1) where $f_{n} \not \equiv 0$ on $A\left(z_{0}, R\right)$. We need only consider the case when $n \geq 1$. 
Since $f$ admits the exceptional value zero at the point $z_{0}$ then from Corollary (1.2) we see that the function $f_{n}$ admits the exceptional value zero at the point $z_{0}$ and the functions $f_{0} / f_{n}, f_{1} / f_{n}, \cdots, f_{n-1} / f_{n}$ have a nonessential singularity at the point $z_{0}$. Now $f-1$ is polyanalytic on $A\left(z_{0}, R\right)$ and admits the exceptional value zero at the point $z_{0}$. Hence the function $\left(f_{0}-1\right) / f_{n}$ has a nonessential singularity at the point $z_{0}$. It now follows that the functions $f_{0}, f_{1}, \cdots, f_{n}$ have a nonessential singularity at the point $z_{0}$. Consequently the polyanalytic function $f$ has a nonessential singularity at the point $z_{0}$. This proves the result.

The above result can be extended if we introduce a more general notion of exceptional value. Let $f$ be polyanalytic with an isolated singularity at the point $z_{0}$. Now let $g$ be polyanalytic with a nonessential isolated singularity at the point $z_{0}$. Then $g$ is said to be an exceptional value for $f$ at $z_{0}$ if and only if $f-g$ has the exceptional value zero at the point $z_{0}$. If $g$ and $h$ are two exceptional values for $f$ at $z_{0}$ these two exceptional values $g$ and $h$ are said to be distinct if and only if $g-h$ does not vanish identically on some annular neighborhood of the point $z_{0}$.

We now have the following general version of the big Picard theorem for polyanalytic functions.

Corollary (4.4). Let $f$ be polyanalytic with an isolated singularity at the point $z_{0}$. If $f$ admits two distinct exceptional values at the point $z_{0}$ then $f$ has a nonessential singularity at the point $z_{0}$.

Proof. Let $g$ and $h$ be the two distinct exceptional values for $f$ at the point $z_{0}$. There is some $0<R \leq+\infty$ so that $f, g$, and $h$ are polyanalytic on $A\left(z_{0}, R\right)$. Assume that $f, g$, and $h$ are represented on $A\left(z_{0}, R\right)$ by means of the equations

$$
f(z)=\sum \bar{z}^{k} f_{k}(z), \quad g(z)=\sum \bar{z}^{k} g_{k}(z), \quad \text { and } \quad b(z)=\sum \bar{z}^{k} b_{k}(z),
$$

$k=0,1, \cdots, n$, where for $k=0,1, \cdots, n$ the functions $f_{k}, g_{k}$, and $h_{k}$ are analytic on $A\left(z_{0}, R\right)$. Since the polyanalytic functions $g$ and $h$ are not identical there is some $j=0,1, \cdots, n$ so that $g_{j} \not h_{j}$. Hence $f_{j} \not g_{j}$ or $f_{j} \not h_{j}$. Assume that $f_{j} \equiv g_{j}$. Since the polyanalytic function $f-g$ admits the exceptional value zero at the point $z_{0}$ we see that $f_{j}-g_{j}$ admits the exceptional value zero at the point $z_{0}$. Also for $i=0,1, \cdots, n$ the functions $\left(f_{i}-g_{i}\right) /\left(f_{j}-g_{j}\right)$ have a nonessential singularity at the point $z_{0}$. Now $f_{j} \equiv h_{j}$ or $f_{j} \equiv h_{j}$. If $f_{j} \equiv h_{j}$ we see that for $i=0,1, \cdots, n$ that the functions $f_{i}$ have a nonessential singularity at the point $z_{0}$. In this case the polyanalytic function $f$ has a nonessential singularity at the point $z_{0}$. Suppose next that $f_{j} \not h_{j}$. Since the polyanalytic function $f-h$ admits the exceptional value zero at the point $z_{0}$ we see that $f_{j}-h_{j}$ admits the exceptional value zero at the point $z_{0}$. In this case we see that $f_{j}$ 
admits the two distinct exceptional values $g_{j}$ and $h_{j}$ at the point $z_{0}$. Hence $f_{j}$ has a nonessential singularity at the point. Thus for $i=0,1, \cdots, n$ we see that the functions $f_{i}$ have a nonessential singularity at the point $z_{0}$. In this case the polyanalytic function has a nonessential singularity at the point $z_{0}$. This proves the result.

The above general version of Picard's big theorem for polyanalytic functions was originally established in [2] by utilizing the theory of quasinormal families of analytic functions and the Poisson-Jensen integral formula.

Note that on the basis of Theorem (1.1) or one of its equivalent formulations in Corollaries (1.1), (1.2), and (1.3), we can obtain a number of results which are similar in character to the result of Corollary (4.4). As one example let $f$ be polyanalytic on $A\left(z_{0}, R\right)$ and represented on $A\left(z_{0}, R\right)$ by means of equation (1.1). Suppose further that there is some $j=0,1, \cdots, n$ such that $f_{j} \equiv 0$ on $A\left(z_{0}, R\right)$ and such that $f_{j}$ has a nonessential isolated singularity at the point $z_{0}$. Then if $f$ admits the exceptional value zero at the point $z_{0}$, it is easy to see that $f$ has a nonessential isolated singularity at the point $z_{0}$. As a further example let $f$ be analytic with an isolated singularity at a point $z_{0}$ and let $P(z, \bar{z})$ be a polynomial in $z$ and $\bar{z}$ of positive degree in $\bar{z}$. Then if $f$ admits the exceptional value $P(z, \bar{z})$ at the point $z_{0}$, it is easy to see that the analytic function $f$ has a nonessential isolated singularity at the point $z_{0}$.

\section{REFERENCES}

1. M. B. Balk, Entire polyanalytic functions with a bounded set of zeros, Izv. Akad. Nauk Armjan. SSR Ser. Mat. 1 (1966), no. 5, 341-357. (Russian) MR 34 \#6127.

2. W. Bosch and P. Krajkiewicz, The big Picard theorem for polyanalytic functions, Proc. Amer. Math. Soc. 26 (1970), 145-150. MR 41 \#8692.

3. H. Cartan, Sur les zéros des combinaisons linéaires de p fonctions holomorphes données, Mathematica (Cluj) 7 (1933), 5-31.

4. T. M. Flett, Note on a function-theoretic identity, J. London Math. Soc. 29 (1954), 115-118. MR 15, 303.

5. W. K. Hayman, Meromorphic functions, Oxford Math. Monographs, Clarendon Press, Oxford, 1964. MR 29\#1337.

6. P. Krajkiewicz, Bianalytic functions with exceptional values, Proc. Amer. Math. Soc. 38 (1973), 75-79.

7. R. Nevanlinna, Eindeutige analytische Funktionen, Die Grundlehren der math. Wissenschaften, Band 46, Springer-Verlag, Berlin, 1936; English transl., Die Grundlehren der math. Wissenschaften, Band 162, Springer-Verlag, Berlin and New York, 1970. MR 43 \#5003.

8. S. Saks and A. Zygmund, Analytic functions: 2nd ed., Monografie Mat., Tom 10, PWN, Warsaw, 1938; English transl., Monografie Mat., Tom 28, PWN, Warsaw, 1965. MR 31 \#4889.

DEPARTMENT OF MATHEMATIÇS, UNIVERSITY OF NEBRASKA, LINCOLN, NEBRASKA 68508 